\title{
Genetic contribution of SCARB1 variants to lipid traits in African Blacks: a candidate gene association study
}

Vipavee Niemsiri ${ }^{1}$, Xingbin Wang ${ }^{1}$, Dilek Pirim ${ }^{1}$, Zaheda H. Radwan' ${ }^{1}$ Clareann H. Bunker ${ }^{2}$, M. Michael Barmada', M. Ilyas Kamboh ${ }^{1 * \dagger}$ and F. Yesim Demirci ${ }^{1^{*} \dagger}$

\begin{abstract}
Background: High-density lipoprotein cholesterol (HDL-C) exerts many anti-atherogenic properties including its role in reverse cholesterol transport (RCT). Scavenger receptor class B member 1 (SCARB1) plays a key role in RCT by selective uptake of HDL cholesteryl esters. We aimed to explore the genetic contribution of SCARB1 to affecting lipid levels in African Blacks from Nigeria.

Methods: We resequenced 13 exons and exon-intron boundaries of SCARB1 in 95 individuals with extreme HDL-C levels using Sanger method. Then, we genotyped 147 selected variants (78 sequence variants, 69 HapMap tagSNPs, and 2 previously reported relevant variants) in the entire sample of 788 African Blacks using either the iPLEX Gold or TaqMan methods. A total of 137 successfully genotyped variants were further evaluated for association with major lipid traits.

Results: The initial gene-based analysis demonstrated evidence of association with HDL-C and apolipoprotein A-I (ApoA-I). The follow-up single-site analysis revealed nominal evidence of novel associations of nine common variants with HDL-C and/or ApoA-I $(P<0.05)$. The strongest association was between rs11057851 and HDL-C $(P=0.0043)$, which remained significant after controlling for multiple testing using false discovery rate. Rare variant association testing revealed a group of 23 rare variants (frequencies $\leq 1 \%)$ associated with HDL-C $(P=0.0478)$. Haplotype analysis identified four SCARB1 regions associated with HDL-C (global $P<0.05$ ).

Conclusions: To our knowledge, this is the first report of a comprehensive association study of SCARB1 variations with lipid traits in an African Black population. Our results showed the consistent association of SCARB1 variants with HDL-C across various association analyses, supporting the role of SCARB1 in lipoprotein-lipid regulatory mechanism.
\end{abstract}

Keywords: African continental ancestry group, Candidate gene association study, Genetic variation, Haplotypes, Lipids, SCARB1 protein, human, Sequence analysis, DNA

\section{Background}

Abnormal lipid and lipoprotein levels are a major risk factor for coronary heart disease (CHD) [1], the leading cause of death worldwide [2]. Elevated low-density lipoprotein cholesterol (LDL-C) levels and decreased high-density lipoprotein cholesterol (HDL-C) levels are

\footnotetext{
*Correspondence: kamboh@pitt.edu; fyd1@pitt.edu

${ }^{\dagger}$ Equal contributors

'Department of Human Genetics, Graduate School of Public Health, University of Pittsburgh, 130 DeSoto Street, Pittsburgh, PA 15261, USA Full list of author information is available at the end of the article
}

correlated with the development of CHD. There is a strong genetic basis for lipoprotein-lipid levels with heritability estimates of $40-80 \%$ [3]. A large number of genes and genetic variants associated with lipid traits have been discovered in genome-wide association studies (GWAS) [4-6]. Most of the common variants (minor allele frequency $[\mathrm{MAF}] \geq 5 \%$ ) identified by GWAS have modest effects on lipid levels, and have overall a small contribution to total genetic variance of lipid traits ( $25-30 \%$ of the heritability) [4-8]. A portion of the missing heritability of lipid traits could be explained by

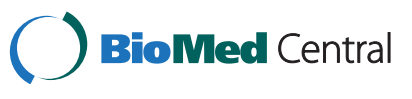

(c) 2015 Niemsiri et al. Open Access This article is distributed under the terms of the Creative Commons Attribution 4.0 International License (http://creativecommons.org/licenses/by/4.0/), which permits unrestricted use, distribution, and reproduction in any medium, provided you give appropriate credit to the original author(s) and the source, provide a link to the Creative Commons license, and indicate if changes were made. The Creative Commons Public Domain Dedication waiver (http://creativecommons.org/publicdomain/zero/1.0/) applies to the data made available in this article, unless otherwise stated. 
low frequency $(\mathrm{LoF}) /$ rare variants $(\mathrm{MAF}<5 \%)$ as suggested by recent studies [9-11].

HDL, the smallest and densest $(d=1.063-1.21 \mathrm{~g} / \mathrm{mL})$ class of lipoprotein particles, has a variety of antiatherogenic properties [12]. One of the HDL properties to protect against CHD is mediated by reverse cholesterol transport (RCT) from peripheral tissues back to the liver [13]. Scavenger receptor class B member 1 (SCARB1, protein; SCARB1, gene) serves as a HDL-C receptor in RCT that mediates selective uptake of HDL-C cholesteryl esters (CE) by the liver and free cholesterol efflux from cells to HDL-C [14]. SCARB1 is also implicated in the metabolism of apolipoprotein $\mathrm{B}$ (ApoB)-containing particles [15-21].

The SCARB1 gene (Entrez Gene ID: 949) is located on human chromosome 12, and is abundantly expressed in liver and steroidogenic tissues [22, 23]. The role of SCARB1 in HDL-C and ApoB-containing lipoproteins metabolism has been established in animal studies. The disruption of SCARB1 is associated with increased HDL$C$ levels and decreased CE uptake [24-26]. Whereas the overexpression of SCARB1 reduces levels of HDL-C, ApoA-I, very low-density lipoprotein cholesterol (VLDLC), LDL-C, and ApoB [15-17, 19] and promotes the hepatic uptake of $\mathrm{CE}$ as well as the biliary secretion of HDL-C [15, 27]. The SCARB1 expression is also significantly associated with hepatic VLDL-triglycerides (TG) and VLDL-ApoB production. Hepatic VLDL cholesterol production together with VLDL clearance is enhanced in response to $S C A R B 1$ overexpression [21]. In contrast, reduced hepatic VLDL-TG and VLDL-ApoB production is associated with SCARB1 knockout status [18, 20, 21].

In humans, three SCARB1 mutations (rs397514572 [p.Ser112Phe], rs187831231 [p.Thr175Ala], and rs387906791 [p.Pro297Ser]; MIM: 601040) have been reported to be associated with significantly increased HDL-C levels [28, 29]. Moreover, several genetic studies have demonstrated the association of common SCARB1 variation with lipoprotein-lipid levels [5, 28-39] and subclinical atherosclerosis [40].

To our knowledge, no genetic study has exclusively investigated the association between SCARB1 and lipid traits in native African populations to date. The objective of this study was to resequence all 13 exons and exon-intron boundaries of SCARB1 in 95 African Blacks from Nigeria with extreme HDL-C levels for variant discovery and then to genotype selected variants in the entire sample of 788 African Blacks, followed by genotype-phenotype association analyses with five major lipid and apolipoprotein (Apo) traits (HDL-C, LDL-C, TG, ApoA-I and ApoB). Because our initial gene-based analysis demonstrated evidence of association with HDL-C and ApoA-I, our subsequent analyses focused on these two traits.

\section{Methods}

\section{Study population}

The present study was carried out on 788 African Black subjects from Benin City, Nigeria, who were recruited as part of a population-based epidemiological study on CHD risk factors. Detailed information on the study design and population description is provided elsewhere [41]. In brief, 788 recruited subjects were healthy civil servants ( $37.18 \%$ females) from three government ministries of the Edo state in Benin City, Nigeria, aged between 19 and 70 years, including 464 junior staff (non-professional staff with salary grades 1-6), and 324 senior staff (professional and administrative staff with salary grades 7-16). The summary features, including biometric and quantitative data of the entire sample of 788 subjects are given in Table 1 and Additional file 1: Table S1.

For resequencing, 95 individuals with extreme HDL$C$ levels (within the upper and lower 10th percentiles of HDL-C distribution) were chosen from the entire sample of 788 African Blacks. Resequencing sample comprised of 48 individuals with high HDL-C levels ( $\geq 90$ th percentile, range $68.30-99.00 \mathrm{mg} / \mathrm{dL}$; Table 1$)$ and 47 individuals with low HDL-C levels ( $\leq 10$ th percentile, range 10.30 $35.00 \mathrm{mg} / \mathrm{dL}$; Table 1). The University of Pittsburgh Institutional Review Board approved the study protocol. All participants gave their informed consent.

\section{Lipid and apolipoprotein measurements}

At least 8-hour fasting blood samples were collected from all participants. Serum specimens were separated by centrifugation of blood samples and then stored at $-70{ }^{\circ} \mathrm{C}$ for $6-12$ months until ready for testing. Lipid and apolipoprotein measurements included total cholesterol, HDL-C, TG, ApoA-I, and ApoB and were done with standard assays at the Heinz Nutrition Laboratory, University of Pittsburgh under the Centers for Disease Control Lipid Standardization Program [41]. LDL-C was calculated with the Friedewald equation [42] when TG levels were less than $400 \mathrm{mg} / \mathrm{dL}$.

\section{PCR and sequencing}

Genomic DNA was isolated from clotted blood using the standard DNA extraction procedure. All 13 SCARB1 exons (isoform 1, NM_005505), exon-intron boundaries, and $1 \mathrm{~kb}$ of each of $5^{\prime}$ and $3^{\prime}$ flanking regions on chromosome 12 (hg19, chr12: 125,262,175-125,348,519) were polymerase chain reaction (PCR) amplified and sequenced. Specific primers were designed using the Primer3 software (Whitehead Institute for Biomedical Research, http://bioinfo.ut.ee/primer3-0.4.0/) to cover 13 target regions, resulting in 14 amplicons, including two overlapping amplicons for the largest last exon 13. PCR reaction and cycling conditions are available upon request. 
Table 1 Characteristics and lipid profile of 95 individuals with extreme ${ }^{a}$ HDL-C levels and of the entire sample of 788 African Blacks

\begin{tabular}{|c|c|c|c|c|}
\hline \multirow{3}{*}{ Variables } & \multicolumn{3}{|c|}{95 Individuals with Extreme ${ }^{a} \mathrm{HDL}-\mathrm{C}$ Levels } & \multirow[t]{3}{*}{ The Entire Sample } \\
\hline & High HDL-C Group & Low HDL-C Group & $P^{d}$ & \\
\hline & (HDL-C range ${ }^{c}: 68.30-99.00 \mathrm{mg} / \mathrm{dL}$ ) & (HDL-C range ${ }^{c}: 10.30-35.00 \mathrm{mg} / \mathrm{dL}$ ) & & \\
\hline $\mathrm{N}($ Females, n) & $48(24)$ & $47(24)$ & 1.00 & $788(293)$ \\
\hline Age, years & $41.29 \pm 8.72$ & $40.87 \pm 7.12$ & 0.80 & $40.95 \pm 8.39$ \\
\hline $\mathrm{BMI}, \mathrm{kg} / \mathrm{m}^{2}$ & $22.06 \pm 4.70$ & $23.91 \pm 5.51$ & 0.08 & $22.87 \pm 4.04$ \\
\hline Total Cholesterol, mg/dL & $201.00 \pm 39.68$ & $141.68 \pm 31.03$ & 2.40E-12 & $172.01 \pm 38.47$ \\
\hline LDL-Cholesterol, mg/dL & $112.55 \pm 39.75$ & $95.04 \pm 28.28$ & 0.02 & $109.25 \pm 34.40$ \\
\hline HDL-Cholesterol, mg/dL & $76.05 \pm 7.53$ & $25.51 \pm 5.66$ & $2.20 \mathrm{E}-16$ & $47.88 \pm 12.87$ \\
\hline Triglycerides, mg/dL & $61.98 \pm 19.85$ & $95.79 \pm 73.21$ & 0.004 & $72.96 \pm 39.32$ \\
\hline Apolipoprotein A-I, mg/dL & $166.04 \pm 28.19$ & $103.84 \pm 27.23$ & $2.20 \mathrm{E}-16$ & $137.03 \pm 28.46$ \\
\hline Apolipoprotein B, mg/dL & $66.00 \pm 20.22$ & $69.64 \pm 21.46$ & 0.40 & $66.98 \pm 22.19$ \\
\hline
\end{tabular}

$B M I$ body mass index, HDL-C/HDL-Cholesterol high-density lipoprotein cholesterol, LDL-Cholesterol low-density lipoprotein cholesterol

Values are presented as unadjusted means \pm standard deviation (SD), unless otherwise mentioned

aDistribution of HDL-C was adjusted for sex and age: HDL-C levels $\geq 90$ th $\%$ tile defined as the "High HDL-C group", and HDL-C levels $\leq 10$ th $\%$ tile defined as the

"Low HDL-C group"

${ }^{\mathrm{b}}$ All data were unadjusted and included individuals with missing values or outliers (values beyond mean \pm 3.5 SD)

'Unadjusted range values

dUnadjusted $P$-values were calculated with t-test or $X^{2}$ test depending on types of variables

The primer sequences and amplicon sizes are given in Additional file 2: Table S2.

Automated DNA sequencing of PCR products was performed in a commercial lab (Beckman Coulter Genomics, Danvers, MA, USA) using Sanger method and ABI 3730XL DNA Analyzers (Applied Biosystems, Waltham, MA, USA). Variant analysis was performed using Variant Reporter (version 1.0, Applied Biosystems, Waltham, MA, USA) and Sequencher (version 4.8, Gene Codes Corporation, Ann Arbor, MI, USA) software in our laboratory.

\section{Variant selection for genotyping}

Of 83 variants identified in the discovery step (see Additional file 3: Table S3, Additional file 4: Table S4, Additional file 5: Figure S1, and Additional file 6: Figure S2), 78 (28 with MAF $\geq 5 \%$ and 50 with MAF $<5 \%$ ) were selected based on the pairwise linkage disequilibrium (LD) and Tagger analysis using an $r^{2}$ threshold of 0.90 (5 were excluded due to high LD) in Haploview (Broad Institute of MIT and Harvard, https://www.broadinstitute.org/scientific-community/science/programs/medical-and-population-genetics/haploview/haploview) [43] for follow-up genotyping in the entire sample $(n=788)$. Since our sequencing was focused primarily on coding regions, in addition we selected 69 HapMap tag single nucleotide polymorphisms [SNPs] (out of total 108 HapMap tagSNPs; see Additional file 7: Table S5 and Additional file 8: Figure S3) based on Tagger analysis (MAF $\geq 5 \%$ and $r^{2} \geq 0.80$ ) of HapMap data (Release \#27) from the Yoruba people of Ibadan, Nigeria (YRI), in order to cover the entire gene for common genetic variation information. Moreover, we selected two SCARB1 variants previously reported to be significantly associated with lipid traits in the literature (Additional file 9: Table S6). Conclusively, a total of 149 variants, comprising of 78 sequence variants, 69 common HapMap-YRI tagSNPs, and two relevant associated variants, were selected for follow-up genotyping.

\section{Genotyping}

Genotyping of selected variants in the total sample of 788 individuals was performed by using either iPLEX Gold (Sequenom, Inc., San Diego, CA, USA) or TaqMan (Applied Biosystems, Waltham, MA, USA) methods and following the manufacturers' protocols.

Out of 149 selected variants, two failed assay designs and nine failed genotyping runs (see details in Additional file 3: Table S3, Additional file 7: Table S5, and Additional file 9: Table S6). Quality control (QC) measures for successfully genotyped variants were as follow: a genotype call rate of $\geq 90 \%$, a discrepancy rate of $<1$ in $10 \%$ replicates, and no deviation from Hardy-Weinberg equilibrium [HWE] $\left(P>3.62 \times 10^{-4}\right.$ after Bonferroni correction). Ultimately, a total of 137 QC-passed genotyped variants were included in genetic association analyses (see Additional file 9: Table S6, Additional file 10: Table S7, Additional file 11: Figure S4, and Additional file 12: Figure S5).

\section{Statistical analysis}

We used the Haploview program to determine allele frequencies, to test HWE for genotype distribution, and to evaluate the LD and pairwise correlations $\left(r^{2}\right)$ between variants [43]. 
The values of each lipid phenotype outside the mean \pm 3.5 standard deviation (SD) were excluded from downstream gene-based, single-site, and haplotype analyses. However, the extreme phenotypic values associated with rare variants (MAF $\leq 1 \%$ ) were maintained during rare variant analysis, as was the case for the p70201/ chr12:125279319 variant (see study workflow in Fig. 1). Values of the five lipid and apolipoprotein traits-HDLC, LDL-C, TG, ApoA-I, and ApoB-were transformed using the Box-Cox transformation. For each trait, we used stepwise regression method to select the most parsimonious set of covariates from the following list: sex, age, body mass index, waist, current smoking (yes/no), minutes of walking or biking to work each day (jobmin), and occupational status (staff: junior [non-professional staff]/senior [professional and administrative staff]). Genetic association analyses, including gene-based, single-site, LoF/rare variant, and haplotype association

\section{Sequencing in a subset of 95 African Blacks with extreme HDL-C levels ( $\leq 10$ th \%tile and $\geq 90$ th \%tile)}

- 83 variants were identified (Table S3)

\section{Genotyping in the entire sample of 788 African Blacks}

- 149 variants were selected:

- 78 of 83 sequence variants (Table S3; 5 were excluded based on LD pattern [see Figures S2 and S3])

- 69 HapMap-YRI tagSNPs (Table S5)

- 2 additional reported lipid-associated variants

\section{Quality control (QC) filtering}

- 137 of 138 successfully genotyped variants passed QC and were advanced into association analyses.

\section{Gene-based association test for 5 traits (HDL-C, LDL-C, TG, ApoA-I, and ApoB)}

- 136 (94 common + 42 LoF/rare*) variants were analyzed

- A significant association with HDL-C and a trend for association with ApoA-I were observed (Table 3)

\section{Association analyses for 2 traits (HDL-C and ApoA-I)}

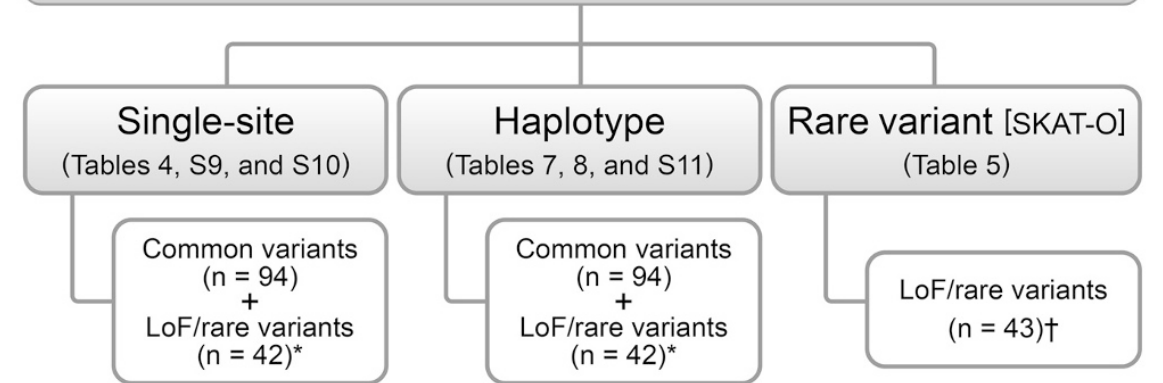

*One variant $(\mathrm{p} 70201 / \mathrm{chr} 12: 125279319, \mathrm{MAF}=0.0010)$ was removed from the analysis due to the exclusion of extreme outlier values (above mean $+3.5 \mathrm{SD}$ ).

†All LoF/rare variants $(n=43)$ were included in this analysis.

Fig. 1 Summary of the study design and flow. Chart presents an overview of the study design and flow, including sequencing and genotyping stages and analysis approaches. ApoA-I, apolipoprotein A-l; ApoB, apolipoprotein B; HDL-C, high-density lipoprotein cholesterol; LD, linkage disequilibrium; LDL-C, low-density lipoprotein cholesterol; LoF, low-frequency; MAF, minor allele frequency; SD, standard deviation; SKAT-O, an optimal sequence kernel association test; SNP, single nucleotide polymorphism; TG, triglycerides; YRI, Yoruba people of Ibadan from Nigeria 
tests, were performed using linear regression models that included significant covariates for each variable (Additional file 13: Table S8).

The gene-based association analysis was conducted under linear additive model for the combined evaluation of common and LoF/rare variants $(n=136$, excluding p70201/ chr12:125279319; see details above in paragraph two of this section) for five major lipid traits using the versatile genebased association study [VEGAS] (http://gump.qimr.edu.au/VEGAS/) software [44]. The significance threshold for the gene-based test was set at $P$-value of 0.05 .

Following gene-based analysis, which primarily implicated SCARB1 in regulation of HDL-C and ApoA-I levels, we further elucidated the association of SCARB1 variants with these two traits using additional tests. In single-site association analysis, $P$-values for each trait were adjusted for multiple testing using BenjaminiHochberg procedure [45] to determine the false discovery rate [FDR] ( $q$-value). For common variants (MAF $\geq 5 \%$ ), a nominal $P$-value of $<0.05$ was considered to be suggestive evidence of association, and an FDR cut-off of 0.20 was used to define statistical significance. For LoF/ rare variants (MAF $<5 \%$ ), the single-site association results were interpreted separately because of inadequate power of our study to detect individual statistical significance for these variants.

We conducted an optimal sequence kernel association test (SKAT-O) [46] to evaluate the association between a total of $43 \mathrm{LoF} /$ rare variants (MAF $<5 \%$ ) and the two lipid traits (HDL-C and ApoA-I) by using three different MAF thresholds: $<5 \%(n=43), \leq 2 \%(n=26)$, and $\leq 1 \%(n=23)$. A significant SKAT-O test was set at a $P$-value of $<0.05$.

Haplotype association analysis was performed using the generalized linear model. We applied a fixed sliding window approach that included four variants per window and sliding for one variant at a time. For each window, a global $P$-value was used to assess the association between the haplotypes with frequency $>1 \%$ and a given trait. A global $P$-value threshold of 0.05 was used to define significant haplotype association.

All analyses, except for VEGAS, were performed using the R statistical software (http://www.r-project.org/) and relevant R packages (i.e., Haplo.Stats for haplotype analysis and SKAT for SKAT-O analysis).

\section{Results}

Identification and distribution of SCARB1 sequence variants in 95 individuals with extreme HDL-C levels

Resequencing of SCARB1 exons and exon-intron boundaries plus flanking regions in 95 African Blacks with extreme HDL-C levels identified 83 variants, of which 51 had MAF $<5 \%$ (Additional file 3: Table S3 and Additional file 5: Figure S1). The majority of 83 variants $(n=73)$ were previously identified (dbSNP build 139: GRCh37.p10).
Most variants $(n=80)$ were singlenucleotide variations [SNVs] (67 transitions and 13 transversions); the rest ( $n=$ 3 ) were short insertion and deletion variations (indels).

Tagger analysis using an $r^{2}$ cutoff of 0.9 identified 28 bins for 32 common variants (MAF $\geq 5 \%$ ), of which three included more than one variant $\left(r^{2}\right.$ ranging from 0.95 to 1.0) (Additional file 6: Figure S2). One of these three bins contained two variants (rs204901986 and rs34339961) in complete LD $\left(r^{2}=1.0\right)$. Of $51 \mathrm{LoF} /$ rare variants (MAF between 1 and $5 \%, n=31$; MAF $\leq 1 \%, n=20), 17$ were present only in the high HDL-C group (MAF ranging between 0.010 and 0.042 ) and eight were observed only in the low HDL-C group (MAF ranging between 0.011 and 0.033). In the high HDL-C group, 29 of $48(\sim 60 \%)$ individuals cumulatively carried at least one LoF/rare variant, ranging from 1 to 7 variants. Similarly, in the low HDL-C group, 27 of $47(\sim 57 \%)$ individuals carried at least one LoF/rare variant, ranging from 1 to 9 variants.

Most variants $(n=60)$ from our sequencing were located in intronic regions, of which two (rs113910315, MAF $=0.005$ and $\mathrm{rs10396210}$, MAF $=0.138$ ) were within splice sites (defined as \pm 20 bp from the start or end of an exon). The former splice site variant was observed only in the low HDL-C group.

Of the total eight coding variants observed, four were common variants (rs2070242 [p.Ser4Ser], rs10396208 [p.Cys21Cys], and rs5888 [p.Ala350Ala], and rs701103 [p.Gly499Arg]-3' untranslated region [UTR] in isoform 1 and exon 13 in isoform 2), and the remaining four were LoF/rare variants (rs4238001 [p.Gly2Ser], rs5891 [p.Val135Ile], rs5892 [p.Phe301Phe], and rs141545424 [p.Gly501Gly]). Of note, two LoF/rare coding variants, (rs5891 [p.Val135Ile] and rs141545424 [p.Gly501Gly]), were found only in the high HDL-C group.

Fifteen variants were located in either UTRs $(n=5)$ or flanking regions $(n=10)$. One 3' UTR variant $(\mathrm{rs} 150512235, \mathrm{MAF}=0.006)$ was very close to a predicted microRNA-145 (miR-145) target site (TargetScanHuman version 6.2, http://www.targetscan.org/). One 5' flanking variant ( $\mathrm{rs} 181338950, \mathrm{MAF}=0.048)$ was located in the putative promoter region [47].

All 10 novel variants (9 SNVs and 1 insertion) identified in this study have been submitted to dbSNP database ([batch ID: SCARB1_AB]:

http://www.ncbi.nlm.nih.gov/SNP/snp_viewTable.cgi?handle $=\mathrm{KAMBOH}$ ) and were non-coding with $\mathrm{MAF}<5 \%$ (ranging between 0.005 and 0.011; Additional file 4: Table S4). Of these novel variants, six and four were present only in the high and low HDL-C groups, respectively.

\section{Genotyping of SCARB1 variants in the entire sample of 788 individuals}

Since our sequencing was focused primarily on coding regions, we selected additional HapMap tagSNPs from the 
HapMap-YRI data in order to cover the entire SCARB1 gene for common genetic variation in SCARB1. Altogether we selected 149 variants for genotyping in our entire African Black sample as follows: 78 variants (28 common variants and $50 \mathrm{LoF} /$ rare variants) discovered in the sequencing step (Additional file 3: Table S3, Additional file 5: Figure S1, and Additional file 6: Figure S2), 69 common HapMap-YRI tagSNPs (Additional file 7: Table S5), and two additional variants with reported association in the literature (Additional file 9: Table S6).

Of these 149 variants, 11 (10 from sequencing, including one promoter [rs181338950], one coding (rs4238001 [p.Gly2Ser]), and one novel [p87459/chr12:125262061], and 1 from HapMap tagSNPs [rs4765180]) failed genotyping, and one (rs866793 from HapMap tagSNPs) failed QC measures. Thus, a total of 137 variants (Additional file 9: Table S6 and Additional file 11: Figure S4) that passed QC were advanced into association analyses with five lipoprotein-lipid traits.

The majority of 137 genotyped variants $(n=120)$ were located in introns, 11 were in exons, and six were in $3^{\prime}$ flanking region (Table 2 and Additional file 12: Figure S5). Ninety-four of 137 variants had MAF $\geq 5 \%$, including four coding variants, one UTR variant, two deletions, and one splice site variant. The remaining 43 variants had MAF $<5 \%$ (MAF between 1 and $5 \%, n=20$; MAF $\leq 1 \%, n=23$ ), including three coding variants, three UTR variants, one insertion, and one splice variant.

Of the 10 novel variants discovered in the sequencing step, nine (8 SNVs and 1 insertion) with MAF $<1 \%$ were successfully genotyped (Additional file 4: Table S4). There was one individual with plasma HDL-C levels above the mean $+3.5 \mathrm{SD}$ carrying one novel variant $-\mathrm{p} 70201 / \mathrm{chr} 12: 125279319$ (MAF $=0.0010)$. Although this extreme HDL-C value was excluded as outlier from the gene-based, single-site, and haplotype analyses, it was included in the SKAT-O rare variant analysis considering a possible large effect size of this variant (Fig. 1).

\section{Gene-based association analyses}

Gene-based tests revealed a nominally significant association $(P=0.0421$; Table 3$)$ of SCARB1 variants with HDL-C levels (best SNP: rs141545424 [p.Gly501Gly], exon 12, $\mathrm{MAF}=0.0007, P=0.0016$ ). Additionally, a trend for association $(P=0.1016)$ was also observed for ApoA-I levels (best SNP: rs7134858, intron 6, MAF = $0.1560, P=0.0052$ ).

Table 2 Distribution of 137 SCARB1 genotyped variants

\begin{tabular}{|c|c|c|c|c|}
\hline & Total & $M A F \geq 5 \%$ & MAF between 1-5\% & MAF $\leq 1 \%$ \\
\hline & N (\%) & n (\%) & n (\%) & n (\%) \\
\hline Total variants & $137(100.00)$ & $94(68.61)$ & $20(14.60)$ & $23(16.79)$ \\
\hline \multicolumn{5}{|l|}{ By known/novel ${ }^{a}$} \\
\hline Known & $128(93.43)$ & $94(68.61)$ & $20(14.60)$ & $14(10.22)$ \\
\hline Single-nucleotide variation & 126 & 92 & 20 & 14 \\
\hline Short indels & 2 & 2 & & \\
\hline Novel & $9(6.57)$ & & & $9(6.57)$ \\
\hline Single-nucleotide variation & 8 & & & 8 \\
\hline Short indels & 1 & & & 1 \\
\hline \multicolumn{5}{|l|}{ By location } \\
\hline Exons-coding ${ }^{c}$ & 7 & $4^{c}$ & 1 & 2 \\
\hline Exons-UTRs & 4 & 1 & 1 & 2 \\
\hline Introns & 118 & 85 & 16 & 17 \\
\hline Introns-splice sites ${ }^{b}$ & 2 & 1 & & 1 \\
\hline 3' flanking & 6 & 3 & 2 & 1 \\
\hline \multicolumn{5}{|l|}{ By amino acid change } \\
\hline Non-synonymous ${ }^{c}$ & 2 & $1^{c}$ & & 1 \\
\hline Synonymous & 5 & 3 & 1 & 1 \\
\hline
\end{tabular}

Indels insertion and deletion variations, MAF minor allele frequency, UTR untranslated region

The list of 137 genotyped variants is shown in Additional file 9: Table S6

The list of 10 novel variants is shown in Additional file 4: Table S4

${ }^{a}$ dbSNP build 139: GRCh37.p10. All 10 novel variants identified in this study have been submitted to dbSNP (batch ID:

SCARB1_AB): http://www.ncbi.nlm.nih.gov/SNP/snp_viewTable.cgi?handle=KAMBOH

${ }^{\mathrm{b}}$ Splice site, defined as $\pm 20 \mathrm{bp}$ from the start or end of an exon

'Including rs701103 (p.Gly499Arg; MAF $=0.2451$ ) that is located in exon 13-3' UTR and translated only in isoform 2 
Table 3 Gene-based association analysis results

\begin{tabular}{|c|c|c|c|c|c|c|}
\hline \multirow[t]{2}{*}{ Trait } & \multirow{2}{*}{$\begin{array}{l}\text { Variants } \\
\text { (n) }\end{array}$} & \multirow{2}{*}{$\begin{array}{l}\text { Test } \\
\text { Statistics }\end{array}$} & \multirow[t]{2}{*}{$P$} & \multicolumn{3}{|l|}{ Best SNP } \\
\hline & & & & SNP Name ${ }^{a}-S N P I^{b}$ & MAF & $P$ \\
\hline $\mathrm{HDL}-\mathrm{C}$ & 136 & 207.5483 & 0.0421 & p82264-rs141545424 & 0.0007 & 0.0016 \\
\hline LDL-C & 136 & 134.1860 & 0.4640 & p32777-rs11057841 & 0.2805 & 0.0047 \\
\hline $\mathrm{TG}$ & 136 & 118.1598 & 0.6700 & p86316-rs701104 & 0.0487 & 0.0357 \\
\hline ApoA-I & 136 & 183.5565 & 0.1016 & p55963-rs7134858 & 0.1560 & 0.0052 \\
\hline ApoB & 136 & 143.7284 & 0.3760 & p22116-rs12370382 & 0.0645 & 0.0153 \\
\hline
\end{tabular}

ApoA-I apolipoprotein A-I, ApoB apolipoprotein B, HDL-C high-density lipoprotein cholesterol, $L D L-C$ low-density lipoprotein cholesterol, MAF minor allele frequency, SNP single nucleotide polymorphism, $T G$ triglycerides

All results were adjusted for covariates: sex, age, body mass index, waist, current smoking (yes/no), minutes of walking or biking to work each day (jobmin), and occupational status [staff: junior (non-professional staff)/senior (professional and administrative staff)]

Nominally significant gene-based $P$-values $(P<0.05)$ are shown in bold

${ }^{a}$ RefSeq of SCARB1: hg19, NM_005505 (CHIP Bioinformatics)

${ }^{b}$ dbSNP build 139: GRCh37.p10

Since the gene-based tests showed evidence of associations with HDL-C and ApoA-I, we primarily focused on these two traits to further examine the SCARB1 variants in the entire sample of 788 African Blacks.

\section{Single-site association analyses of common SCARB1 variants}

Of 94 common SCARB1 variants with MAF $\geq 5 \%, 10$ showed nominal associations $(P<0.05)$ with HDL-C and/ or ApoA-I (Table 4; see results for each trait in Additional file 14: Table S9 and Additional file 15: Table S10), of which three (rs11057851, rs4765615, and rs838895) exhibited associations with both HDL-C and ApoA-I.

The most significant association was found between rs11057851 and HDL-C $(\beta=-0.5924, P=0.0043$, FDR $=$ 0.1465). The second best association was between rs7134858 and ApoA-I $(\beta=1.7537, P=0.0052$, FDR $=$ 0.2918 ), followed by the association of rs5888 (p.Ala350Ala) with ApoA-I $(\beta=2.0962, P=0.0080, \mathrm{FDR}=0.2918)$.

Of 10 variants that showed nominal associations, high LD $\left(r^{2}>0.80\right)$ was observed for two pairs of variants (Fig. 2), between rs8388912 and rs5888 (p.Ala350Ala; $\left.r^{2}=0.86\right)$, and between rs838896 and rs838895 $\left(r^{2}=0.84\right)$.

\section{Association analyses of low-frequency/rare SCARB1 variants}

The LoF/rare variants $(n=43)$ were categorized into three groups based on their frequencies for association analysis with HDL-C and ApoA-I using SKAT-O: MAF $<5 \%(n=43), \operatorname{MAF} \leq 2 \%(n=26)$, and MAF $\leq 1 \%(n=$ $23)$. Although no association between LoF/rare variants and ApoA-I was detected, the group of 23 variants with MAF $\leq 1 \%$ yielded nominal association with HDL-C levels $(P=0.0478$; Table 5$)$.

We then individually examined the association of 23 variants with MAF $\leq 1 \%$ with HDL-C and ApoA-I. Six of these rare variants showed association with either HDL-C levels or both HDL-C and ApoA-I levels (Table 6). While three of them are known variants (rs115604379, rs377124254, and rs141545424 [p.Gly501Gly]), the other three are novel (p52919/chr12:125296601, p54611/chr12:125294909, and p54856/chr12:125294664). Moreover, four of these six rare variants (rs377124254, rs141545424 [p.Gly501Gly], p54611/chr12:125294909, and p54856/chr12:125294664) were present in individuals with extreme phenotypic values (above or below the 3rd percentile). Two of these variants (rs377124254: $\beta=11.5518, P=0.0016$; rs141545424 [p.Gly501Gly]: $\quad \beta=11.585, \quad P=0.0016)$ were found in a single subject who had very high HDL-C level. Whereas the other two were observed in one individual each, who had extremely low HDL$C$ levels (p54611/chr12:125294909: $\beta=-9.5243, P=0.0097$; p54856/chr12:125294664: $\quad \beta=-8.4305, \quad P=0.0215)$ and ApoA-I levels (p54611/chr12:125294909: $\beta=-19.3821, P=$ 0.0344; p54856/chr12:125294664: $\beta=-24.0757, P=0.0082$ ). This rare variant group also included a novel variant (p70201/chr12:125279319) that was observed in one individual with an unusually high plasma HDL-C level (above the mean $+3.5 \mathrm{SD}$ ).

\section{Haplotype association analyses}

The 4-SNP sliding window haplotype analyses revealed associations of 32 haplotype windows with HDL-C and/ or ApoA-I (global $P<0.05$; Table 7; see results for each trait in Additional file 16: Table S11), of which five (windows \#47, \#72, \#111, \#112, and \#123) were associated with both.

Overall, a total of 21 haplotype windows showed significant associations with ApoA-I, of which 10 contained seven variants associated with ApoA-I in single-site analysis. Haplotype window \#110 spanning introns 10-11 showed the best association signal (global $P=0.0012$ ) and contained the rs838896 variant with a nominal evidence of association with ApoA-I $(P=0.0278)$ in single-site analysis. 
Table 4 Nominally significant single-site associations $(P<0.05)$ of common SCARB1 variants

\begin{tabular}{|c|c|c|c|c|c|c|c|c|c|c|c|c|c|c|}
\hline $\begin{array}{l}\text { SNP } \\
\text { Name }^{a} \\
\end{array}$ & SNP ID & $\begin{array}{l}\text { Chr12 } \\
\text { Position }^{c} \\
\end{array}$ & Location & $\begin{array}{l}\text { Amino Acid } \\
\text { Change }\end{array}$ & $\begin{array}{l}\text { RegDB } \\
\text { Score }^{d}\end{array}$ & $\begin{array}{l}\text { Major/Minor } \\
\text { Alleles }\end{array}$ & MAF & $\beta$ & SE & $R^{2}(\%)$ & $P$ & FDR & $\begin{array}{l}\text { Secondary Trait } \\
\text { (Effect) }\end{array}$ & $\begin{array}{l}\text { Top } 3 \\
\text { Variants }\end{array}$ \\
\hline \multicolumn{15}{|l|}{ HDL-C } \\
\hline p20207 & rs11057853 & 125329313 & Intron 1 & & 5 & $\mathrm{G} / \mathrm{A}$ & 0.4484 & 0.4082 & 0.1925 & 1.0650 & 0.0343 & 0.4235 & & \\
\hline p20741 & rs11057851 & 125328779 & Intron 1 & & 5 & $\mathrm{C} / \mathrm{T}$ & 0.3237 & -0.5924 & 0.2067 & 1.3010 & 0.0043 & 0.1465 & ApoA-I $(\downarrow)$ & Top 1 \\
\hline p45516 & rs1902569 & 125304004 & Intron 1 & & 5 & $\mathrm{G} / \mathrm{A}$ & 0.1544 & 0.5447 & 0.2629 & 0.6390 & 0.0386 & 0.4375 & & \\
\hline p49690 & rs4765615 & 125299830 & Intron 2 & & 5 & $\mathrm{G} / \mathrm{A}$ & 0.4426 & -0.4646 & 0.1866 & 0.9330 & 0.0130 & 0.2526 & ApoA-I $(\downarrow)$ & \\
\hline p79828 & rs838895 & 125269692 & Intron 11 & & 5 & $C / G$ & 0.3171 & 0.4961 & 0.2059 & 0.8220 & 0.0162 & 0.2756 & ApoA-I ( $\uparrow)$ & \\
\hline \multicolumn{15}{|l|}{ ApoA-I } \\
\hline p20741 & rs11057851 & 125328779 & Intron 1 & & 5 & $C / T$ & 0.3237 & -1.2331 & 0.5117 & 0.8600 & 0.0162 & 0.3186 & $\mathrm{HDL}-\mathrm{C}(\downarrow)$ & \\
\hline p49690 & rs4765615 & 125299830 & Intron 2 & & 5 & $\mathrm{G} / \mathrm{A}$ & 0.4426 & -0.9139 & 0.4614 & 0.6770 & 0.0480 & 0.5022 & HDL-C ( $\downarrow)$ & \\
\hline p55963 & rs7134858 & 125293557 & Intron 6 & & 6 & $\mathrm{C} / \mathrm{T}$ & 0.1560 & 1.7537 & 0.6260 & 1.0710 & 0.0052 & 0.2918 & & Top 2 \\
\hline p63483 & rs838912 & 125286037 & Intron 7 & & 7 & $\mathrm{G} / \mathrm{A}$ & 0.0867 & 1.8700 & 0.8230 & 0.6880 & 0.0234 & 0.3972 & & \\
\hline p64772 & rs5888 & 125284748 & Exon 8 & Ala350Ala & $3 a$ & $C / T$ & 0.0961 & 2.0962 & 0.7888 & 0.9460 & 0.0080 & 0.2918 & & Top 3 \\
\hline p79721 & rs838896 & 125269799 & Intron 11 & & 5 & $\mathrm{G} / \mathrm{C}$ & 0.3104 & 1.1147 & 0.5056 & 0.7270 & 0.0278 & 0.4197 & & \\
\hline p79828 & rs838895 & 125269692 & Intron 11 & & 5 & $C / G$ & 0.3171 & 1.2206 & 0.5074 & 0.7800 & 0.0164 & 0.3186 & HDL-C ( $(\uparrow)$ & \\
\hline p83884 & rs701106 & 125265636 & Intron 12 & & 5 & $\mathrm{C} / \mathrm{T}$ & 0.2597 & 1.2967 & 0.5352 & 0.7770 & 0.0156 & 0.3186 & & \\
\hline
\end{tabular}

ApoA-I apolipoprotein A-I, FDR false discovery rate, HDL-C high-density lipoprotein cholesterol, MAF minor allele frequency, RegDB RegulomeDB, SE standard error, SNP single nucleotide polymorphism, UTR untranslated region, $R^{2}$, the proportion of the phenotypic variance explained by the variant; $\downarrow$, decreased; $\uparrow$, increased

Alleles on reverse strand. HDL-C and ApoA-I variables were in mg/dL and Box-Cox transformed

Results were adjusted for covariats: sex, age, waist, current smoking (yes/no), and minutes of walking or biking to work each day (jobmin) for HDL-C; sex and age for ApoA-I

The most significant $P$-value for each trait is shown in bold, see the single-site association $\left(-\log _{10} P\right)$ plot and pairwise correlations $\left(r^{2}\right)$ in Fig. 2

FDR that reached a threshold of $<0.20$ is shown in bold

a, cRefSeq of SCARB1: hg19, NM_005505 (CHIP Bioinformatics)

bdbSNP build 139: GRCh37.p10

${ }^{\mathrm{d}}$ Detailed RegulomeDB (version 1.0) scoring scheme is described in Additional file 17: Table S12 or at http://regulome.stanford.edu/help, see functional assignments in Additional file 18: Table S13 


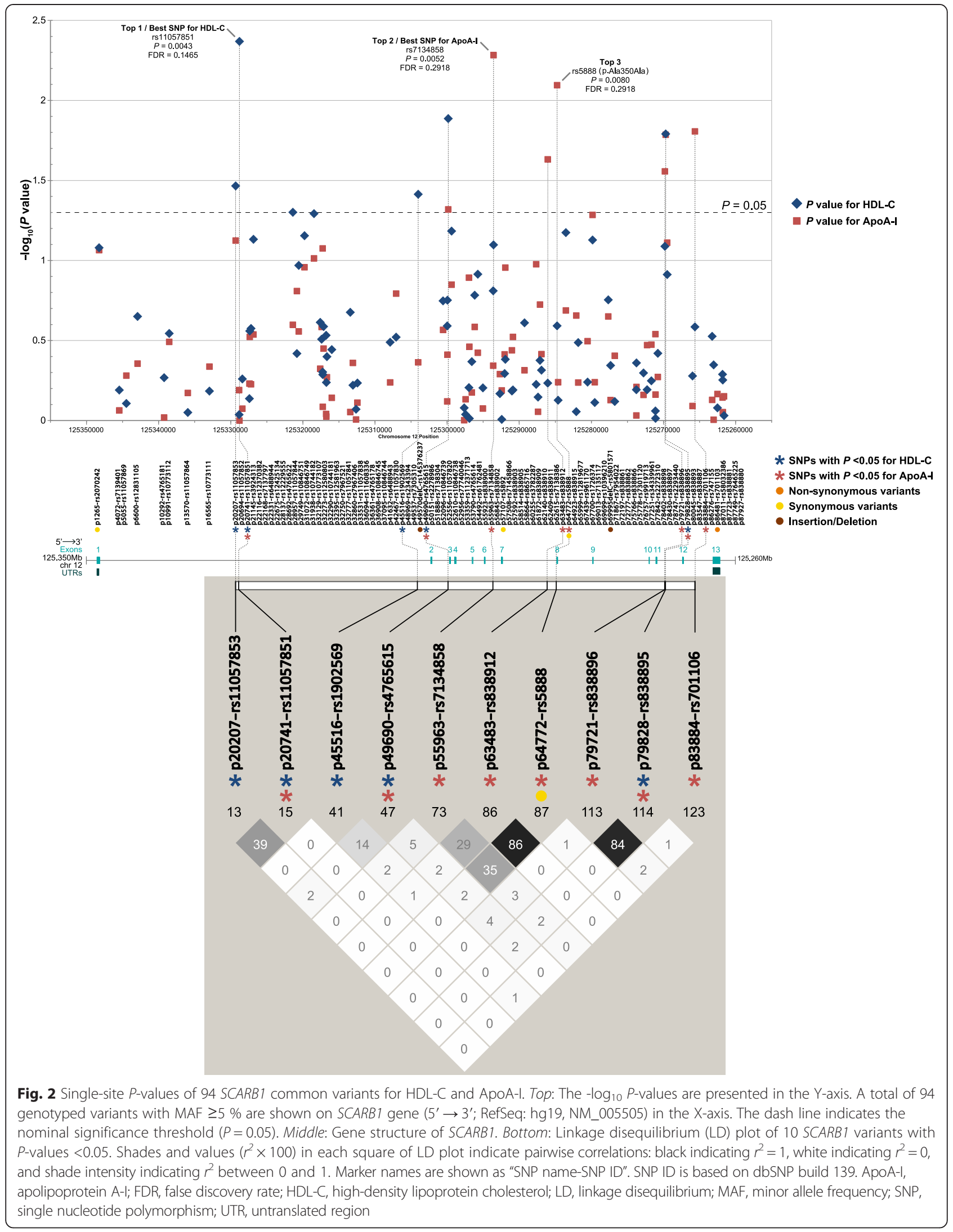


Table 5 Association results for low-frequency and rare SCARB1 variants (MAF $<5 \%)$

\begin{tabular}{|c|c|c|c|c|c|c|}
\hline \multirow[t]{2}{*}{ MAF } & \multirow{2}{*}{$\begin{array}{l}\text { No of } \\
\text { Variants }\end{array}$} & \multirow{2}{*}{$\begin{array}{l}\text { No of Samples with/ } \\
\text { without Variants }\end{array}$} & \multicolumn{2}{|l|}{$\mathrm{HDL}-\mathrm{C}$} & \multicolumn{2}{|l|}{ ApoA-I } \\
\hline & & & Stat & $P$ & Stat & $P$ \\
\hline$\leq 0.01$ & $23^{a}$ & $93 / 694$ & 126653.8207 & 0.0478 & 60151.0985 & 0.3707 \\
\hline$\leq 0.02$ & 26 & $134 / 653$ & 123009.0805 & 0.1324 & 48439.6697 & 0.5166 \\
\hline$<0.05$ & 43 & $442 / 346$ & 135697.1974 & 0.0737 & 298813.0544 & 0.1517 \\
\hline
\end{tabular}

ApoA-I apolipoprotein A-I, HDL-C high-density lipoprotein cholesterol, MAF minor allele frequency, SD standard deviation, SNP single nucleotide polymorphism Results were adjusted for covariates: sex, age, waist, current smoking (yes/no), and minutes of walking or biking to work each day (jobmin) for HDL-C; sex and age for ApoA-I

Nominally significant $P$-values $(P<0.05)$ are shown in bold

ancluding p70201/chr12:125279319 that was observed in one individual with an outlier value (above the mean +3.5 SD). See details in Result Section 3.5

A total of 16 haplotype windows yielded significant associations with HDL-C, of which seven contained three HDL-C-associated variants detected in single-site analysis. The most significant association was found with window \#111 (global $P=0.0040$ ) spanning intron 11 , which contained the rs838895 variant nominally associated with HDL-C $(P=0.0162)$ in single-site analysis.

We observed nine regions (5 regions for ApoA-I and 4 regions for $\mathrm{HDL}-\mathrm{C}$ ) harboring consecutive significant haplotype windows (global $P<0.05$; ranging from 2 to 6 windows per region; Table 8 and Fig. 3). Seven of those regions contained at least one of the six variants that exhibited nominal associations $(P<0.05)$ with HDL-C and/ or ApoA-I (rs4765615, rs7134858, rs838912, rs838896, rs838895, and rs701106) in single-site analysis.

\section{Functional evaluation of identified variants}

In order to examine the possible regulatory function of all 153 SCARB1 variants (83 variants identified by our sequencing, 68 common HapMap tagSNPs [excluding rs4765180 due to genotyping failure; see Additional file 7: Table S5], and two relevant variants from the literature), we used the RegulomeDB database (version 1.0, Stanford University, http://www.regulomedb.org/) [48]. Although most of 153 variants $(n=132)$ revealed scores ranging from 1 to 6 , only 11 were supported by strong evidence for regulatory function (scores of $1 f-2 b$ ): one promoter, one 5' UTR, two coding (rs2070242 [p.Ser4Ser] and rs10396208 [p.Cys21Cys]), five intronic, one 3' UTR, and one 3' flanking variants. Summary and detailed regulatory functions are provided in Additional file 17: Table S12 and Additional file 18: Table S13.

Of 10 variants associated with HDL-C and/or ApoA-I, only one ApoA-I associated variant (rs5888 [p.Ala350Ala] in exon 8) showed suggestive evidence of regulatory function with a score of 3a (Table 4).

Of 10 novel variants, one insertion variant (p1048insC/ chr12:125348472) located in 5' UTR-exon 1 had a strong potential for regulatory function with a score of 2a (Additional file 4: Table S4).
Comparison of SCARB1 single-site and haplotype association analysis results between African Blacks (this study) and US Non-Hispanic Whites (previous study [49])

We compared SCARB1 single-site and haplotype association results in African Blacks reported in this study to those in US Non-Hispanic Whites (NHWs) reported in our previously published study [49]. In the sequencing stage, the number of variants identified in African Blacks $(n=83)$ was greater than that in US NHWs $(n=44)$. Notably, most ( $90 \%)$ of the 22 sequence variants that were shared between the two populations differed in minor alleles and/or MAFs. Although our major findings included the associations with HDL-C and ApoA-I in African Blacks, we also sought to replicate four associations observed with ApoB levels in US NHWs [49] (Table 9); the association between rs11057820 and ApoB $(P<0.05)$ that we previously reported in US NHWs [49] was also observed in African Blacks (US NHWs [G allele]: $\beta=0.8700, P=0.0436$; African Blacks [A allele]: $\beta=1.8661, P=0.0292$ ). In addition, we observed two variants (rs4765615 and rs701106) exhibiting nominal associations $(P<0.05)$ in both populations, albeit with different lipid traits (US NHWs| rs4765615 [G allele]: $\beta$ $=1.2493, P=0.0059$ for ApoB; rs701106 [T allele]: $\beta=$ 0.0394, $P=0.0066$ for HDL-C; African Blacks rs4765615 [A allele]: $\beta=-0.4646, P=0.013$ for HDL-C and $\beta=-0.9139, P=0.048$ for ApoA-I; rs701106 [T allele]: $\beta=1.2967, P=0.0156$ for ApoA-I). Moreover, we noticed that two regions associated with HDL-C or ApoA-I (global $P<0.05$; Table 10) in African Blacks spanning intron 2 and intron 3 overlapped with the ApoB-associated region (Region I in Fig. 4) previously reported in US NHWs [49]. Three haplotype regions associated with HDL-C (global $P<0.05$ ) spanning intron 11 and exon 13-3' UTR in African Blacks also overlapped with a large HDL-C-associated region (Region II in Fig. 4) previously reported in US NHWs [49].

\section{Discussion}

Our sequencing identified 83 variants, of which 78 were selected for follow-up genotyping in the total sample of 
Table 6 Characteristics and effects of 6 SCARB1 rare variants of interest

\begin{tabular}{|c|c|c|c|c|c|c|c|c|c|c|c|c|c|c|c|c|}
\hline SNP Name ${ }^{a}$ & SNP ID & Chr12 Position ${ }^{c}$ & Location & $\begin{array}{l}\text { Amino } \\
\text { Acid } \\
\text { Change }\end{array}$ & RegDB Score ${ }^{d}$ & $\begin{array}{l}\text { Major/Minor } \\
\text { Alleles }\end{array}$ & MAF & GT & $\begin{array}{l}\text { GT Count } \\
\text { (Carrier Freq) }\end{array}$ & $\begin{array}{l}\text { Adjusted } \\
\text { Mean } \pm \mathrm{SD} \\
(\mathrm{mg} / \mathrm{dL})\end{array}$ & $\beta$ & SE & $R^{2}(\%)$ & $P$ & FDR & $\begin{array}{l}\text { Second } \\
\text { Assoc Trait } \\
\text { (Effect) }\end{array}$ \\
\hline \multicolumn{17}{|l|}{ HDL-C } \\
\hline \multirow[t]{2}{*}{ p52919 } & & 125296601 & Intron 4 & & 5 & $\mathrm{G} / \mathrm{T}$ & 0.0013 & GG & 734 & $47.87 \pm 12.71$ & -7.4063 & 2.5863 & 1.1050 & 0.0043 & 0.1465 & ApoA-I ( $\downarrow)$ \\
\hline & & & & & & & & GT & $2(0.27)$ & $24.67 \pm 9.26$ & & & & & & \\
\hline \multirow[t]{2}{*}{ p53372 } & rs115604379 & 125296148 & Intron 5 & & 5 & $C / T$ & 0.0066 & CC & 729 & $47.68 \pm 12.64$ & 3.0372 & 1.1642 & 0.9140 & 0.0093 & 0.2190 & \\
\hline & & & & & & & & $\mathrm{CT}$ & $10(1.35)$ & $58.2 \pm 13.03$ & & & & & & \\
\hline \multirow[t]{2}{*}{ p54611 } & & 125294909 & Intron 5 & & 4 & $\mathrm{~T} / \mathrm{C}$ & 0.0007 & $\pi$ & 742 & $47.86 \pm 12.68$ & -9.5243 & 3.6710 & 0.8920 & 0.0097 & 0.2190 & ApoA-I $(\downarrow)$ \\
\hline & & & & & & & & TC & $1(0.13)$ & $19.59 \pm \mathrm{NA}$ & & & & & & \\
\hline \multirow[t]{2}{*}{ p54856 } & & 125294664 & Intron 6 & & 4 & $C / T$ & 0.0007 & CC & 742 & $47.85 \pm 12.70$ & -8.4305 & 3.6579 & 0.7130 & 0.0215 & 0.3243 & ApoA-I ( $\downarrow)$ \\
\hline & & & & & & & & $C T$ & $1(0.13)$ & $21.48 \pm N A$ & & & & & & \\
\hline \multirow[t]{2}{*}{ p77620 } & rs377124254 & 125271900 & Intron 10 & & 5 & $\mathrm{G} / \mathrm{A}$ & 0.0007 & GG & 735 & $47.77 \pm 12.67$ & 11.5518 & 3.6514 & 1.3500 & 0.0016 & 0.1104 & \\
\hline & & & & & & & & GA & $1(0.14)$ & $90.2 \pm N A$ & & & & & & \\
\hline \multirow[t]{2}{*}{ p82264 } & rs141545424 & 125267256 & Exon 12 & Gly501Gly & 5 & $C / A$ & 0.0007 & CC & 739 & $47.77 \pm 12.66$ & 11.5850 & 3.6469 & 1.3530 & 0.0016 & 0.1104 & \\
\hline & & & & & & & & CA & $1(0.14)$ & $90.31 \pm N A$ & & & & & & \\
\hline \multicolumn{17}{|l|}{ ApoA-I } \\
\hline \multirow[t]{2}{*}{ p52919 } & & 125296601 & Intron 4 & & 5 & $\mathrm{G} / \mathrm{T}$ & 0.0013 & GG & 741 & $136.81 \pm 27.74$ & -13.4137 & 6.4689 & 0.5750 & 0.0385 & 0.4359 & HDL-C ( $\downarrow)$ \\
\hline & & & & & & & & GT & $2(0.27)$ & $97.42 \pm 18.38$ & & & & & & \\
\hline \multirow[t]{2}{*}{ p54611 } & & 125294909 & Intron 5 & & 4 & $\mathrm{~T} / \mathrm{C}$ & 0.0007 & $\pi$ & 748 & $136.83 \pm 27.66$ & -19.2831 & 9.0970 & 0.5980 & 0.0344 & 0.4359 & HDL-C ( $\downarrow)$ \\
\hline & & & & & & & & TC & $1(0.13)$ & $80.62 \pm N A$ & & & & & & \\
\hline \multirow[t]{2}{*}{ p54856 } & & 125294664 & Intron 6 & & 4 & $C / T$ & 0.0007 & CC & 748 & $136.87 \pm 27.61$ & -24.0757 & 9.0781 & 0.9330 & 0.0082 & 0.2918 & HDL-C ( () \\
\hline & & & & & & & & $\mathrm{CT}$ & $1(0.13)$ & $67.98 \pm N A$ & & & & & & \\
\hline
\end{tabular}

ApoA-I apolipoprotein A-I, FDR false discovery rate, GT genotype, HDL-C high-density lipoprotein cholesterol, MAF minor allele frequency, RegDB RegulomeDB, SD standard deviation, SE standard error, SNP single nucleotide polymorphism; $R^{2}$, the proportion of the phenotypic variance explained by the variant; $\downarrow$, decreased

All alleles were on reverse stand. HDL-C and ApoA-I variables were in $\mathrm{mg} / \mathrm{dL}$ and Box-Cox transformed

Results were adjusted for covariates: sex, age, waist, current smoking (yes/no), and minutes of walking or biking to work each day (jobmin) for HDL-C; sex and age for ApoA-I.

Detailed single-site association results are shown in Additional file 14: Table S9 and Additional file 15: Table S10.

a, cRefSeq of SCARB1: hg19, NM_005505 (CHIP Bioinformatics)

bdbSNP build 139: GRCh37.p10. All 10 novel variants identified in this study have been submitted to dbSNP (batch ID: SCARB1 AB): http://www.ncbi.nIm.nih.gov/SNP/snp_viewTable.cgi?handle=KAMBOH

${ }^{\mathrm{d}}$ The RegulomeDB (version 1.0) scoring scheme and functional assignments are described in Additional file 17: Table S12 and Additional file 18: Table S13, respectively 
Table 7 Significant haplotype association (global $P<0.05$ ) of 136 SCARB1 genotyped variants with HDL-C and ApoA-I

\begin{tabular}{|c|c|c|c|c|c|c|c|c|c|c|c|c|c|c|c|c|}
\hline \multirow{2}{*}{$\begin{array}{l}\text { Wind } \\
\text { \# }\end{array}$} & SNP 1 - SNP 4 & \multirow{2}{*}{$\begin{array}{l}\text { Chr12 } \\
\text { Position }\end{array}$} & \multirow[t]{2}{*}{ Location } & \multirow{2}{*}{$\begin{array}{l}\text { Amino } \\
\text { Acid } \\
\text { Change }\end{array}$} & \multirow{2}{*}{$\begin{array}{l}\text { Major/ } \\
\text { Minor } \\
\text { Alleles }\end{array}$} & \multirow[t]{2}{*}{ MAF } & \multirow[t]{2}{*}{$\beta$} & \multirow{2}{*}{$\begin{array}{l}\text { Single- } \\
\text { site } P\end{array}$} & \multirow[t]{2}{*}{ Haplotype \# } & \multirow{2}{*}{$\begin{array}{l}\text { Hap } \\
\text { Seq }\end{array}$} & \multirow{2}{*}{$\begin{array}{l}\text { Hap } \\
\text { Freq }\end{array}$} & \multirow[t]{2}{*}{ Coef } & \multirow[t]{2}{*}{ SE } & \multirow[t]{2}{*}{ t.stat } & \multirow[t]{2}{*}{ Hap $P$} & \multirow{2}{*}{$\begin{array}{l}\text { Global } \\
P\end{array}$} \\
\hline & $\begin{array}{l}\text { (SNP Name } \text { S-SNP }^{\mathrm{a} N \mathrm{P}} \\
\text { ID }^{\mathrm{b}} / \mathrm{Ch}^{\mathrm{c}} \text { ) }\end{array}$ & & & & & & & & & & & & & & & \\
\hline \multicolumn{17}{|c|}{$\mathrm{HDL}-\mathrm{C}$} \\
\hline 39 & p41632-rs6488943 & 125307888 & Intron 1 & & $A / C$ & 0.2954 & -0.2195 & 0.3244 & h39.1 & CCGG & 0.0315 & 0.4305 & 0.6471 & 0.6654 & 0.5060 & 0.0207 \\
\hline 39 & p42467-rs11057830 & 125307053 & Intron 1 & & $C / T$ & 0.1523 & -0.2810 & 0.3015 & h39.2 & CCGA & 0.2508 & -0.5918 & 0.2725 & -2.1713 & 0.0302 & \\
\hline 39 & p45516-rs1902569 & 125304004 & Intron 1 & & $\mathrm{G} / \mathrm{A}$ & 0.1544 & 0.5447 & 0.0386 & h39.3 & ATGA & 0.1414 & -0.6841 & 0.3192 & -2.1433 & 0.0324 & \\
\hline \multirow[t]{4}{*}{39} & p45627-rs12297372 & 125303893 & Intron 1 & & $A / G$ & 0.0487 & -0.0483 & 0.9156 & h39.4 & ACAA & 0.1514 & 0.1991 & 0.2963 & 0.6720 & 0.5018 & \\
\hline & & & & & & & & & h39.5 & ACGG & 0.0155 & -1.7144 & 0.9080 & -1.8880 & 0.0594 & \\
\hline & & & & & & & & & h39.6 (rare) & $* * * *$ & 0.0148 & 2.5239 & 1.0902 & 2.3151 & 0.0209 & \\
\hline & & & & & & & & & hap.base39 & ACGA & 0.3946 & NA & NA & NA & NA & \\
\hline 44 & p48969-rs2343394 & 125300551 & Intron 2 & & $C / T$ & 0.1898 & 0.3165 & 0.1788 & h44.1 & TCWG & 0.1855 & 0.5292 & 0.2523 & 2.0977 & 0.0363 & 0.0271 \\
\hline 44 & p49537-rs7305310 & 125299983 & Intron 2 & & $C / T$ & 0.1007 & -0.3396 & 0.2566 & h44.2 & CCDG & 0.2244 & 0.4676 & 0.2429 & 1.9249 & 0.0546 & \\
\hline 44 & p49570delC-rs145376237 & 125299950 & Intron 2 & & W/D & 0.2276 & 0.3121 & 0.1773 & h44.3 & CCWG & 0.0446 & 1.0491 & 0.4882 & 2.1489 & 0.0320 & \\
\hline \multirow[t]{3}{*}{44} & p49690-rs4765615 & 125299830 & Intron 2 & & $\mathrm{G} / \mathrm{A}$ & 0.4426 & -0.4646 & 0.0130 & h44.4 & CTWG & 0.1018 & -0.1197 & 0.3121 & -0.3835 & 0.7015 & \\
\hline & & & & & & & & & h44.5 (rare) & $* * * *$ & 0.0089 & -0.9887 & 1.0998 & -0.8990 & 0.3689 & \\
\hline & & & & & & & & & hap.base44 & CCWA & 0.4348 & NA & NA & NA & NA & \\
\hline 45 & p49537-rs7305310 & 125299983 & Intron 2 & & $C / T$ & 0.1007 & -0.3396 & 0.2566 & h45.1 & CDGC & 0.2282 & 0.4661 & 0.2393 & 1.9473 & 0.0519 & 0.0155 \\
\hline 45 & p49570delC-rs145376237 & 125299950 & Intron 2 & & W/D & 0.2276 & 0.3121 & 0.1773 & h45.2 & CWGC & 0.2302 & 0.6926 & 0.2376 & 2.9146 & 0.0037 & \\
\hline 45 & p49690-rs4765615 & 125299830 & Intron 2 & & $\mathrm{G} / \mathrm{A}$ & 0.4426 & -0.4646 & 0.0130 & h45.3 & TWGC & 0.1020 & -0.0653 & 0.3085 & -0.2115 & 0.8325 & \\
\hline \multirow[t]{2}{*}{45} & p49759-rs146272788 & 125299761 & Intron 2 & & $C / T$ & 0.0020 & 2.5988 & 0.2219 & h45.4 (rare) & $* * * *$ & 0.0030 & 2.0667 & 2.0848 & 0.9913 & 0.3219 & \\
\hline & & & & & & & & & hap.base45 & CWAC & 0.4366 & NA & NA & NA & NA & \\
\hline 46 & p49570delC-rs145376237 & 125299950 & Intron 2 & & W/D & 0.2276 & 0.3121 & 0.1773 & h46.1 & DGCG & 0.2228 & 0.4373 & 0.2413 & 1.8123 & 0.0703 & 0.0278 \\
\hline 46 & p49690-rs4765615 & 125299830 & Intron 2 & & $\mathrm{G} / \mathrm{A}$ & 0.4426 & -0.4646 & 0.0130 & h46.2 & WGCG & 0.3311 & 0.4910 & 0.2105 & 2.3326 & 0.0199 & \\
\hline 46 & p49759-rs146272788 & 125299761 & Intron 2 & & $C / T$ & 0.0020 & 2.5988 & 0.2219 & h46.3 (rare) & $* * * *$ & 0.0080 & 1.9089 & 1.0569 & 1.8061 & 0.0713 & \\
\hline 46 & p49978-rs5891 & 125299542 & Exon 3 & Val135lle & $\mathrm{G} / \mathrm{A}$ & 0.0058 & 1.3374 & 0.2791 & hap.base46 & WACG & 0.4381 & NA & NA & NA & NA & \\
\hline 47 & p49690-rs4765615 & 125299830 & Intron 2 & & $\mathrm{G} / \mathrm{A}$ & 0.4426 & -0.4646 & 0.0130 & h47.1 & ACGG & 0.4346 & -0.4701 & 0.1824 & -2.5777 & 0.0101 & 0.0079 \\
\hline 47 & p49759-rs146272788 & 125299761 & Intron 2 & & $C / T$ & 0.0020 & 2.5988 & 0.2219 & h47.2 (rare) & $* * * *$ & 0.0101 & 1.4683 & 0.9441 & 1.5552 & 0.1203 & \\
\hline 47 & p49978-rs5891 & 125299542 & Exon 3 & Val135lle & $\mathrm{G} / \mathrm{A}$ & 0.0058 & 1.3374 & 0.2791 & hap.base47 & GCGG & 0.5553 & NA & NA & NA & NA & \\
\hline 47 & p50024-rs368880622 & 125299496 & Intron 3 & & $\mathrm{G} / \mathrm{T}$ & 0.0026 & 1.6506 & 0.4362 & & & & & & & & \\
\hline 63 & p53359-rs112371713 & 125296161 & Intron 5 & & $\mathrm{G} / \mathrm{A}$ & 0.1243 & 0.4193 & 0.1651 & h63.1 & ACGA & 0.1237 & 0.3273 & 0.3011 & 1.0871 & 0.2773 & 0.0394 \\
\hline 63 & p53372-rs115604379 & 125296148 & Intron 5 & & $C / T$ & 0.0066 & 3.0372 & 0.0093 & h63.2 & GCGG & 0.0427 & -0.1630 & 0.4738 & -0.3441 & 0.7309 & \\
\hline 63 & p53790-rs4765614 & 125295730 & Intron 5 & & $\mathrm{G} / \mathrm{A}$ & 0.2653 & -0.3281 & 0.1218 & h63.3 & GCAA & 0.2678 & -0.2408 & 0.2194 & -1.0975 & 0.2728 & \\
\hline
\end{tabular}


Table 7 Significant haplotype association (global $P<0.05)$ of 136 SCARB1 genotyped variants with HDL-C and ApoA-I (Continued)

\begin{tabular}{llll}
\hline $\mathbf{6 3}$ & $\mathrm{p} 544445-\mathrm{r} 600910935$ & 125295075 & Intron 5 \\
& & & \\
$\mathbf{7 2}$ & $\mathrm{p} 55923-\mathrm{r} 8389000$ & 125293597 & Intron 6 \\
$\mathbf{7 2}$ & $\mathrm{p} 55963-\mathrm{rs} 7134858$ & 125293557 & Intron 6 \\
$\mathbf{7 2}$ & $\mathrm{p} 56845$-rs838902 & 125292675 & Intron 6 \\
$\mathbf{7 2}$ & $\mathrm{p} 57004-\mathrm{rs} 187562853$ & 125292516 & Intron 6
\end{tabular}

$\begin{array}{llll}\mathbf{1 1 1} & \text { p78747-rs2293440 } & 125270773 & \text { Intron 11 } \\ \mathbf{1 1 1} & \text { p78791-rs75289200 } & 125270729 & \text { Intron 11 } \\ \mathbf{1 1 1} & \text { p79721-rs838896 } & 125269799 & \text { Intron 11 } \\ \mathbf{1 1 1} & \text { p79828-rs838895 } & 125269692 & \text { Intron 11 }\end{array}$

112 p78791-rs75289200

125270729 Intron 11

112 p79721-rs838896

125269799 Intron 11

112 p79828-rs838895

112 p $80045-r s 838893$

125269692 Intron 11

125269475 Intron 11

$\begin{array}{llll}\mathbf{1 1 3} & \text { p79721-rs838896 } & 125269799 & \text { Intron } 11 \\ \mathbf{1 1 3} & \text { p79828-rs838895 } & 125269692 & \text { Intron 11 } \\ \mathbf{1 1 3} & \text { p80045-rs838893 } & 125269475 & \text { Intron 11 } \\ \mathbf{1 1 3} & \text { p81863-rs185445624 } & 125267657 & \text { Intron 11 }\end{array}$

114 p79828-rs838895

125269692 Intron 11

114 p 80045 -rs 838893

125269475 Intron 11

$\begin{array}{lllll}\mathrm{A} / \mathrm{G} & 0.0418 & -0.1247 & 0.7963 & \mathrm{~h} 63 \\ \mathrm{G} / \mathrm{A} & 0.3921 & 0.2787 & 0.1549 & \mathrm{~h} 72 \\ \mathrm{C} / \mathrm{T} & 0.1560 & 0.4418 & 0.0799 & \mathrm{~h} 72 \\ \mathrm{~A} / \mathrm{G} & 0.4249 & -0.0786 & 0.6801 & \mathrm{~h} 72 \\ \mathrm{G} / \mathrm{A} & 0.0098 & 1.6474 & 0.0872 & \mathrm{~h} 72\end{array}$

63.4 (rare)

hap.base63 GCGA 0.5591 NA NA NA NA

$\begin{array}{llllllll}\text { h72.1 } & \text { ACAG } & 0.2725 & 0.4039 & 0.2520 & 1.6024 & 0.1095 & 0.0315\end{array}$

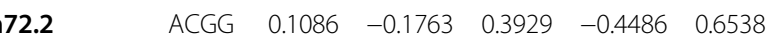

$\begin{array}{lllllll}\text { h72.3 } & \text { GTAG } & 0.1284 & 0.3877 & 0.3170 & 1.2228 & 0.2218\end{array}$

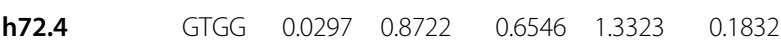

h72.5 GCAG $0.1716 \quad-0.4913 \quad 0.3344 \quad-1.4690 \quad 0.1422$

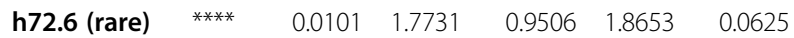

hap.base72 GCGG 0.2791 NA NA NA NA

$\begin{array}{llllllllllll}\mathrm{T} / \mathrm{C} & 0.4112 & -0.1684 & 0.3806 & \mathbf{h} 111.1 & \text { CCCG } & 0.0306 & 0.7458 & 0.5599 & 1.3321 & 0.1832 & 0.0040\end{array}$

$\begin{array}{llllllllllll}\text { T/C } & 0.0321 & 0.7037 & 0.2078 & \mathbf{h} 111.2 & \text { CTGC } & 0.1534 & -0.5556 & 0.2830 & -1.9629 & 0.0500\end{array}$

$\begin{array}{lllllllllll}\text { G/C } & 0.3104 & 0.3565 & 0.0817 & \mathbf{h} 111.3 & \text { CTCG } & 0.2269 & 0.1234 & 0.2391 & 0.5162 & 0.6058\end{array}$

$\begin{array}{lllllllllll}\mathrm{C} / \mathrm{G} & 0.3171 & 0.4961 & \mathbf{0 . 0 1 6 2} & \mathbf{h} 111.4 & \text { TTGG } & 0.0180 & 2.3022 & 0.7617 & 3.0225 & 0.0026\end{array}$

$\begin{array}{lllllll}\text { h111.5 } & \text { TTCG } & 0.0439 & 0.5755 & 0.5317 & 1.0823 & 0.2795\end{array}$

$\begin{array}{lllllll}\text { h111.6 } & \text { TTCC } & 0.0145 & 0.9606 & 0.8068 & 1.1907 & 0.2342\end{array}$

h111.7 (rare) $\quad * * * * \quad 0.0033 \quad 0.7755 \quad 2.1917 \quad 0.3538 \quad 0.7236$

hap.base111 TTGC 0.5094 NA NA NA NA

$\begin{array}{llllllllllll}\mathrm{T} / \mathrm{C} & 0.0321 & 0.7037 & 0.2078 & \mathbf{h} 1 \mathbf{1 2 . 1} & \text { CCGA } & 0.0311 & 0.7440 & 0.5559 & 1.3384 & 0.1812 & 0.0055\end{array}$

$\begin{array}{lllllllllll}\text { G/C } & 0.3104 & 0.3565 & 0.0817 & \text { h112.2 } & \text { TGGA } & 0.0171 & 2.3734 & 0.7506 & 3.1621 & 0.0016\end{array}$

$\begin{array}{llllllllllll}\text { C/G } & 0.3171 & 0.4961 & \mathbf{0 . 0 1 6 2} & \mathbf{h} 1 \mathbf{1 2 . 3} & \text { TGCA } & 0.0112 & -1.2672 & 0.9074 & -1.3964 & 0.1630\end{array}$

$\begin{array}{lllllllllll}\text { G/A } & 0.3244 & 0.3127 & 0.1224 & \mathbf{h} 112.4 & \text { TCGA } & 0.2704 & 0.2488 & 0.2164 & 1.1501 & 0.2505\end{array}$

$\begin{array}{lllllll}\text { h112.5 } & \text { TCCG } & 0.0139 & 1.1219 & 0.8186 & 1.3704 & 0.1710\end{array}$

h112.6 (rare) $\quad{ }^{* * * *} \quad \begin{array}{llllll}0.0068 & 1.6244 & 1.2691 & 1.2800 & 0.2009\end{array}$

hap.base112 TGCG 0.6493 NA NA NA NA

$\begin{array}{llllllllllll}\text { G/C } & 0.3104 & 0.3565 & 0.0817 & \mathbf{h 1 1 3 . 1} & \text { GGAG } & 0.0171 & 2.3949 & 0.7509 & 3.1895 & 0.0015 & 0.0048\end{array}$

$\begin{array}{llllllllllll}\text { C/G } & 0.3171 & 0.4961 & \mathbf{0 . 0 1 6 2} & \mathbf{h} 1 \mathbf{1 3 . 2} & \text { GCAG } & 0.0120 & -1.1963 & 0.8784 & -1.3619 & 0.1736\end{array}$

$\begin{array}{lllllllllll}\text { G/A } & 0.3244 & 0.3127 & 0.1224 & \mathbf{h} 113.3 & \text { CGAG } & 0.2996 & 0.3071 & 0.2067 & 1.4861 & 0.1377\end{array}$

$\begin{array}{llllllllllll}\text { G/A } & 0.0020 & -0.9612 & 0.6510 & \mathbf{h} 113.4 & \text { CCGG } & 0.0139 & 1.1509 & 0.8168 & 1.4090 & 0.1592\end{array}$

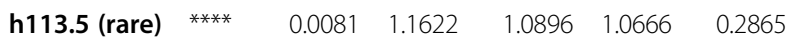

hap.base113 GCGG 0.6493 NA NA NA NA

$\begin{array}{llllllllllll}C / G & 0.3171 & 0.4961 & \mathbf{0 . 0 1 6 2} & \mathbf{h} 1 \mathbf{1 4 . 1} & \text { GAGC } & 0.3173 & 0.3755 & 0.2023 & 1.8559 & 0.0639 & 0.0447\end{array}$

$\begin{array}{llllllllllll}\mathrm{G} / \mathrm{A} & 0.3244 & 0.3127 & 0.1224 & \mathbf{h} 114.2 & \text { CGGT } & 0.0306 & -0.8840 & 0.5344 & -1.6541 & 0.0985\end{array}$ 
Table 7 Significant haplotype association (global $P<0.05)$ of 136 SCARB1 genotyped variants with HDL-C and ApoA-I (Continued)

\begin{tabular}{|c|c|c|c|c|c|c|c|c|c|c|c|c|c|c|c|c|}
\hline 114 & p81863-rs185445624 & 125267657 & Intron 11 & & $\mathrm{G} / \mathrm{A}$ & 0.0020 & -0.9612 & 0.6510 & h114.3 & CAGC & 0.0111 & -1.2612 & 0.9170 & -1.3754 & 0.1694 & \\
\hline \multirow[t]{2}{*}{114} & p82019-rs838890 & 125267501 & Intron 11 & & $\mathrm{C} / \mathrm{T}$ & 0.0320 & -1.0051 & 0.0618 & h114.4 (rare) & $* * * *$ & 0.0086 & 0.9073 & 1.0936 & 0.8296 & 0.4070 & \\
\hline & & & & & & & & & hap.base114 & CGGC & 0.6325 & NA & NA & NA & NA & \\
\hline 117 & p82019-rs838890 & 125267501 & Intron 11 & & $\mathrm{C} / \mathrm{T}$ & 0.0320 & -1.0051 & 0.0618 & h117.1 & CCAG & 0.0238 & -1.0596 & 0.6275 & -1.6884 & 0.0917 & 0.0433 \\
\hline 117 & p82264-rs141545424 & 125267256 & Exon 12 & Gly501Gly & $C / A$ & 0.0007 & 11.5850 & 0.0016 & h117.2 & TCGG & 0.0311 & -0.9657 & 0.5302 & -1.8215 & 0.0689 & \\
\hline 117 & p82340-rs77483223 & 125267180 & Intron 12 & & $\mathrm{G} / \mathrm{A}$ & 0.0231 & -1.0458 & 0.1012 & h117.3 (rare) & $* * * *$ & 0.0067 & 1.6191 & 1.2946 & 1.2507 & 0.2114 & \\
\hline 117 & p82369-rs75446635 & 125267151 & Intron 12 & & $\mathrm{G} / \mathrm{A}$ & 0.0059 & 0.5896 & 0.6322 & hap.base117 & CCGG & 0.9383 & NA & NA & NA & NA & \\
\hline 118 & p82264-rs141545424 & 125267256 & Exon 12 & Gly501Gly & $C / A$ & 0.0007 & 11.5850 & 0.0016 & h118.1 & CAGT & 0.0238 & -1.0621 & 0.6274 & -1.6929 & 0.0909 & 0.0375 \\
\hline 118 & p82340-rs77483223 & 125267180 & Intron 12 & & $\mathrm{G} / \mathrm{A}$ & 0.0231 & -1.0458 & 0.1012 & h118.2 & CGGC & 0.0307 & -1.0134 & 0.5313 & -1.9073 & 0.0569 & \\
\hline 118 & p82369-rs75446635 & 125267151 & Intron 12 & & $\mathrm{G} / \mathrm{A}$ & 0.0059 & 0.5896 & 0.6322 & h118.3 (rare) & $* * * *$ & 0.0067 & 1.6189 & 1.2762 & 1.2685 & 0.2050 & \\
\hline 118 & p82434-rs838889 & 125267086 & Intron 12 & & $\mathrm{~T} / \mathrm{C}$ & 0.0315 & -1.0389 & 0.0526 & hap.base118 & CGGT & 0.9387 & NA & NA & NA & NA & \\
\hline 123 & p83884-rs701106 & 125265636 & Intron 12 & & $\mathrm{C} / \mathrm{T}$ & 0.2597 & 0.2471 & 0.2601 & h123.1 & TCCT & 0.0256 & -1.2114 & 0.6218 & -1.9483 & 0.0518 & 0.0386 \\
\hline 123 & p86245-rs188375019 & 125263275 & Intron 12 & & $\mathrm{C} / \mathrm{T}$ & 0.0341 & 0.7447 & 0.1639 & h123.2 & TCCG & 0.2327 & 0.5306 & 0.2403 & 2.2085 & 0.0275 & \\
\hline 123 & p86276-rs747155 & 125263244 & Intron 12 & & $\mathrm{C} / \mathrm{T}$ & 0.1495 & 0.2793 & 0.2980 & h123.3 & CCTG & 0.1476 & 0.3955 & 0.2811 & 1.4071 & 0.1598 & \\
\hline \multirow[t]{4}{*}{123} & p86316-rs701104 & 125263204 & Intron 12 & & $\mathrm{G} / \mathrm{T}$ & 0.0487 & -0.9838 & 0.0286 & h123.4 & CCCT & 0.0233 & -0.2329 & 0.7038 & -0.3309 & 0.7408 & \\
\hline & & & & & & & & & h123.5 & CTCG & 0.0330 & 0.8888 & 0.5458 & 1.6283 & 0.1039 & \\
\hline & & & & & & & & & h123.6 (rare) & $* * * *$ & 0.0029 & 1.1191 & 3.2961 & 0.3395 & 0.7343 & \\
\hline & & & & & & & & & hap.base123 & CCCG & 0.5348 & NA & NA & NA & NA & \\
\hline 124 & p86245-rs188375019 & 125263275 & Intron 12 & & $\mathrm{C} / \mathrm{T}$ & 0.0341 & 0.7447 & 0.1639 & h124.1 & CTGA & 0.1476 & 0.1530 & 0.2692 & 0.5683 & 0.5700 & 0.0368 \\
\hline 124 & p86276-rs747155 & 125263244 & Intron 12 & & $\mathrm{C} / \mathrm{T}$ & 0.1495 & 0.2793 & 0.2980 & h124.2 & CCTG & 0.0465 & -1.1879 & 0.4699 & -2.5281 & 0.0117 & \\
\hline 124 & p86316-rs701104 & 125263204 & Intron 12 & & $\mathrm{G} / \mathrm{T}$ & 0.0487 & -0.9838 & 0.0286 & h124.3 & CCGA & 0.0915 & 0.1086 & 0.3376 & 0.3218 & 0.7477 & \\
\hline \multirow[t]{3}{*}{124} & p86481-rs701103 & 125263039 & Exon 13-3' UTR & $\begin{array}{l}\text { Gly499Arg } \\
\text { (isoform 2) }\end{array}$ & $\mathrm{G} / \mathrm{A}$ & 0.2451 & 0.1642 & 0.4492 & h124.4 & TCGG & 0.0337 & 0.7348 & 0.5362 & 1.3702 & 0.1710 & \\
\hline & & & & & & & & & h124.5 (rare) & $* * * *$ & 0.0045 & 4.0859 & 2.1131 & 1.9336 & 0.0535 & \\
\hline & & & & & & & & & hap.base124 & CCGG & 0.6761 & NA & NA & NA & NA & \\
\hline 125 & p86276-rs747155 & 125263244 & Intron 12 & & $C / T$ & 0.1495 & 0.2793 & 0.2980 & h125.1 & TGAA & 0.1476 & 0.1543 & 0.2689 & 0.5737 & 0.5664 & 0.0307 \\
\hline 125 & p86316-rs701104 & 125263204 & Intron 12 & & $\mathrm{G} / \mathrm{T}$ & 0.0487 & -0.9838 & 0.0286 & h125.2 & CTGA & 0.0465 & -1.1980 & 0.4691 & -2.5535 & 0.0109 & \\
\hline 125 & p86481-rs701103 & 125263039 & Exon 13-I3' UTR & $\begin{array}{l}\text { Gly499Arg } \\
\text { (isoform 2) }\end{array}$ & $\mathrm{G} / \mathrm{A}$ & 0.2451 & 0.1642 & 0.4492 & h125.3 & CGAA & 0.0915 & 0.1139 & 0.3375 & 0.3375 & 0.7359 & \\
\hline \multirow[t]{2}{*}{125} & p86967-rs187492239 & 125262553 & Exon 13-3' UTR & & $A / G$ & 0.0355 & 0.7743 & 0.1412 & h125.4 & CGGG & 0.0352 & 0.7974 & 0.5241 & 1.5216 & 0.1285 & \\
\hline & & & & & & & & & h125.5 (rare) & $* * * *$ & 0.0045 & 4.0989 & 2.1134 & 1.9394 & 0.0528 & \\
\hline
\end{tabular}


Table 7 Significant haplotype association (global $P<0.05$ ) of 136 SCARB1 genotyped variants with HDL-C and ApoA-I (Continued)

\begin{tabular}{|c|c|c|c|c|c|c|c|c|c|c|c|c|c|c|c|c|}
\hline & & & & & & & & & hap.base125 & CGGA & 0.6747 & NA & NA & NA & NA & \\
\hline \multicolumn{17}{|c|}{ ApoA-I } \\
\hline 47 & p49690-rs4765615 & 125299830 & Intron 2 & & $\mathrm{G} / \mathrm{A}$ & 0.4426 & -0.9139 & 0.0480 & h47.1 & ACGG & 0.4351 & -0.8907 & 0.4584 & -1.9432 & 0.0524 & 0.0343 \\
\hline 47 & p49759-rs146272788 & 125299761 & Intron 2 & & $C / T$ & 0.0020 & 1.5883 & 0.7630 & h47.2 (rare) & $* * * *$ & 0.0106 & 3.5858 & 2.2998 & 1.5592 & 0.1194 & \\
\hline 47 & p49978-rs5891 & 125299542 & Exon 3 & Val135lle & $\mathrm{G} / \mathrm{A}$ & 0.0058 & 5.6762 & 0.0628 & hap.base47 & GCGG & 0.5543 & NA & NA & NA & NA & \\
\hline 47 & p50024-rs368880622 & 125299496 & Intron 3 & & $\mathrm{G} / \mathrm{T}$ & 0.0026 & 1.6012 & 0.7255 & & & & & & & & \\
\hline 48 & p49759-rs146272788 & 125299761 & Intron 2 & & $C / T$ & 0.0020 & 1.5883 & 0.7630 & h48.1 & CGGT & 0.0206 & 3.3555 & 1.6564 & 2.0258 & 0.0431 & 0.0293 \\
\hline 48 & p49978-rs5891 & 125299542 & Exon 3 & Val135lle & $\mathrm{G} / \mathrm{A}$ & 0.0058 & 5.6762 & 0.0628 & h48.2 (rare) & $* * * *$ & 0.0106 & 4.0750 & 2.3644 & 1.7235 & 0.0852 & \\
\hline 48 & p50024-rs368880622 & 125299496 & Intron 3 & & $\mathrm{G} / \mathrm{T}$ & 0.0026 & 1.6012 & 0.7255 & hap.base48 & CGGC & 0.9688 & NA & NA & NA & NA & \\
\hline 48 & p50118-rs58710319 & 125299402 & Intron 3 & & $C / T$ & 0.0208 & 3.1376 & 0.0571 & & & & & & & & \\
\hline 49 & p49978-rs5891 & 125299542 & Exon 3 & Val135lle & $\mathrm{G} / \mathrm{A}$ & 0.0058 & 5.6762 & 0.0628 & h49.1 & GGTT & 0.0213 & 3.3792 & 1.6416 & 2.0584 & 0.0399 & 0.0289 \\
\hline 49 & p50024-rs368880622 & 125299496 & Intron 3 & & $\mathrm{G} / \mathrm{T}$ & 0.0026 & 1.6012 & 0.7255 & h49.2 & GGCC & 0.1928 & 0.8864 & 0.5841 & 1.5176 & 0.1295 & \\
\hline 49 & p50118-rs58710319 & 125299402 & Intron 3 & & $C / T$ & 0.0208 & 3.1376 & 0.0571 & h49.3 (rare) & $* * * *$ & 0.0086 & 4.7388 & 3.1873 & 1.4868 & 0.1375 & \\
\hline 49 & p50151-rs2278986 & 125299369 & Intron 3 & & $\mathrm{~T} / \mathrm{C}$ & 0.1933 & 0.8568 & 0.1419 & hap.base 49 & $\mathrm{GGCT}$ & 0.7774 & NA & NA & NA & NA & \\
\hline 70 & p54627-chr12_125294893 & 125294893 & Intron 5 & & $\mathrm{G} / \mathrm{C}$ & 0.0020 & 3.6910 & 0.4850 & h70.1 & GCAC & 0.3873 & 0.8579 & 0.5090 & 1.6854 & 0.0923 & 0.0140 \\
\hline 70 & p54856-chr12_125294664 & 125294664 & Intron 6 & & $C / T$ & 0.0007 & -24.0757 & 0.0082 & h70.2 & GCGT & 0.1568 & 2.0940 & 0.6700 & 3.1254 & 0.0018 & \\
\hline 70 & p55923-rs838900 & 125293597 & Intron 6 & & $\mathrm{G} / \mathrm{A}$ & 0.3921 & 0.3606 & 0.4549 & h70.3 (rare) & $* * * *$ & 0.0027 & -2.5567 & 5.2200 & -0.4898 & 0.6244 & \\
\hline 70 & p55963-rs7134858 & 125293557 & Intron 6 & & $\mathrm{C} / \mathrm{T}$ & 0.1560 & 1.7537 & 0.0052 & hap.base70 & GCGC & 0.4532 & NA & NA & NA & NA & \\
\hline 71 & p54856-chr12_125294664 & 125294664 & Intron 6 & & $C / T$ & 0.0007 & -24.0757 & 0.0082 & h71.1 & CACA & 0.2736 & 0.7883 & 0.6210 & 1.2694 & 0.2047 & 0.0488 \\
\hline 71 & p55923-rs838900 & 125293597 & Intron 6 & & $\mathrm{G} / \mathrm{A}$ & 0.3921 & 0.3606 & 0.4549 & h71.2 & CACG & 0.1134 & 1.1284 & 0.9724 & 1.1604 & 0.2462 & \\
\hline 71 & p55963-rs7134858 & 125293557 & Intron 6 & & $C / T$ & 0.1560 & 1.7537 & 0.0052 & h71.3 & CGTA & 0.1296 & 2.1103 & 0.7906 & 2.6691 & 0.0078 & \\
\hline \multirow[t]{3}{*}{71} & p56845-rs838902 & 125292675 & Intron 6 & & $\mathrm{~A} / \mathrm{G}$ & 0.4249 & -0.3052 & 0.5129 & h71.4 & CGTG & 0.0300 & 2.1358 & 1.6772 & 1.2734 & 0.2032 & \\
\hline & & & & & & & & & h71.5 & CGCA & 0.1706 & -0.1013 & 0.8355 & -0.1212 & 0.9035 & \\
\hline & & & & & & & & & hap.base71 & CGCG & 0.2822 & NA & NA & NA & NA & \\
\hline 72 & p55923-rs838900 & 125293597 & Intron 6 & & $\mathrm{G} / \mathrm{A}$ & 0.3921 & 0.3606 & 0.4549 & h72.1 & ACAG & 0.2733 & 0.7471 & 0.6218 & 1.2016 & 0.2299 & 0.0463 \\
\hline 72 & p55963-rs7134858 & 125293557 & Intron 6 & & $\mathrm{C} / \mathrm{T}$ & 0.1560 & 1.7537 & 0.0052 & h72.2 & ACGG & 0.1057 & 0.7094 & 0.9850 & 0.7202 & 0.4716 & \\
\hline 72 & p56845-rs838902 & 125292675 & Intron 6 & & $\mathrm{~A} / \mathrm{G}$ & 0.4249 & -0.3052 & 0.5129 & h72.3 & GTAG & 0.1297 & 2.0304 & 0.7898 & 2.5707 & 0.0103 & \\
\hline \multirow[t]{4}{*}{72} & p57004-rs187562853 & 125292516 & Intron 6 & & $\mathrm{G} / \mathrm{A}$ & 0.0098 & 3.2853 & 0.1690 & h72.4 & GTGG & 0.0299 & 2.1741 & 1.6857 & 1.2897 & 0.1975 & \\
\hline & & & & & & & & & h72.5 & GCAG & 0.1712 & -0.3122 & 0.8263 & -0.3778 & 0.7057 & \\
\hline & & & & & & & & & h72.6 (rare) & $* * * *$ & 0.0100 & 3.9105 & 2.4373 & 1.6044 & 0.1090 & \\
\hline & & & & & & & & & hap.base72 & GCGG & 0.2801 & NA & NA & NA & NA & \\
\hline 78 & p57592-rs838903 & 125291928 & Intron 7 & & $\mathrm{G} / \mathrm{A}$ & 0.3763 & -0.7661 & 0.1109 & h78.1 & GCAC & 0.0559 & 1.8913 & 1.0469 & 1.8067 & 0.0712 & 0.0326 \\
\hline
\end{tabular}


Table 7 Significant haplotype association (global $P<0.05$ ) of 136 SCARB1 genotyped variants with HDL-C and ApoA-I (Continued)

\begin{tabular}{|c|c|c|c|c|c|c|c|c|c|c|c|c|c|c|c|}
\hline 78 & p58514-rs838905 & 125291006 & Intron 7 & $\mathrm{~T} / \mathrm{C}$ & 0.4329 & -0.4213 & 0.3646 & h78.2 & GTAC & 0.0367 & 1.0784 & 1.2814 & 0.8415 & 0.4003 & \\
\hline 78 & p58664-rs865716 & 125290856 & Intron 7 & $A / T$ & 0.2708 & 0.5369 & 0.3008 & h78.3 & GTAT & 0.2557 & 0.3365 & 0.6035 & 0.5576 & 0.5773 & \\
\hline \multirow[t]{4}{*}{78} & p60255-rs3782287 & 125289265 & Intron 7 & $C / T$ & 0.2831 & 0.3715 & 0.4856 & h78.4 & GTTC & 0.2463 & 0.4962 & 0.5864 & 0.8462 & 0.3977 & \\
\hline & & & & & & & & h78.5 & $\mathrm{GTT}$ & 0.0238 & 5.5715 & 1.6643 & 3.3477 & 0.0009 & \\
\hline & & & & & & & & h78.6 (rare) & $* * * *$ & 0.0075 & 0.6333 & 2.9303 & 0.2161 & 0.8289 & \\
\hline & & & & & & & & hap.base78 & ACAC & 0.3740 & NA & NA & NA & NA & \\
\hline 79 & p58514-rs838905 & 125291006 & Intron 7 & $\mathrm{~T} / \mathrm{C}$ & 0.4329 & -0.4213 & 0.3646 & h79.1 & CACT & 0.1270 & 0.3290 & 0.8318 & 0.3955 & 0.6926 & 0.0256 \\
\hline 79 & p58664-rs865716 & 125290856 & Intron 7 & $A / T$ & 0.2708 & 0.5369 & 0.3008 & h79.2 & TACC & 0.0379 & 0.6384 & 1.2921 & 0.4941 & 0.6214 & \\
\hline 79 & p60255-rs3782287 & 125289265 & Intron 7 & $C / T$ & 0.2831 & 0.3715 & 0.4856 & h79.3 & TATC & 0.2563 & 0.1851 & 0.6336 & 0.2921 & 0.7703 & \\
\hline \multirow[t]{5}{*}{79} & p61872-rs838909 & 125287648 & Intron 7 & $C / T$ & 0.2199 & 0.9232 & 0.1056 & h79.4 & TTCC & 0.1587 & -0.6020 & 0.7769 & -0.7749 & 0.4386 & \\
\hline & & & & & & & & h79.5 & TTCT & 0.0880 & 1.8902 & 0.8856 & 2.1342 & 0.0331 & \\
\hline & & & & & & & & h79.6 & TाTC & 0.0238 & 5.1755 & 1.6851 & 3.0714 & 0.0022 & \\
\hline & & & & & & & & h79.7 (rare) & $* * * *$ & 0.0059 & 1.2466 & 3.1079 & 0.4011 & 0.6885 & \\
\hline & & & & & & & & hap.base79 & CACC & 0.3024 & NA & NA & NA & NA & \\
\hline 80 & p58664-rs865716 & 125290856 & Intron 7 & $A / T$ & 0.2708 & 0.5369 & 0.3008 & h80.1 & ACCG & 0.0389 & -0.3521 & 1.2793 & -0.2753 & 0.7832 & 0.0030 \\
\hline 80 & p60255-rs3782287 & 125289265 & Intron 7 & $C / T$ & 0.2831 & 0.3715 & 0.4856 & h80.2 & ACTG & 0.1274 & -0.1816 & 0.7909 & -0.2297 & 0.8184 & \\
\hline 80 & p61872-rs838909 & 125287648 & Intron 7 & $C / T$ & 0.2199 & 0.9232 & 0.1056 & h80.3 & ATCG & 0.2611 & -0.1400 & 0.6323 & -0.2213 & 0.8249 & \\
\hline \multirow[t]{5}{*}{80} & p62140-rs838910 & 125287380 & Intron 7 & $\mathrm{G} / \mathrm{T}$ & 0.3047 & -0.0755 & 0.8821 & h80.4 & TCCG & 0.1549 & -1.3614 & 0.7489 & -1.8178 & 0.0695 & \\
\hline & & & & & & & & h80.5 & TCTG & 0.0901 & 2.0511 & 0.8921 & 2.2992 & 0.0218 & \\
\hline & & & & & & & & h80.6 & TTCG & 0.0224 & 4.7307 & 1.8842 & 2.5107 & 0.0123 & \\
\hline & & & & & & & & h80.7 (rare) & $* * * *$ & 0.0083 & 3.1429 & 3.4362 & 0.9147 & 0.3607 & \\
\hline & & & & & & & & hap.base80 & ACCT & 0.2970 & NA & NA & NA & NA & \\
\hline 81 & p60255-rs3782287 & 125289265 & Intron 7 & $C / T$ & 0.2831 & 0.3715 & 0.4856 & h81.1 & CCGC & 0.1740 & -1.5355 & 0.7276 & -2.1103 & 0.0352 & 0.0050 \\
\hline 81 & p61872-rs838909 & 125287648 & Intron 7 & $C / T$ & 0.2199 & 0.9232 & 0.1056 & h81.2 & CCGT & 0.0215 & -0.5623 & 1.6155 & -0.3481 & 0.7279 & \\
\hline 81 & p62140-rs838910 & 125287380 & Intron 7 & $\mathrm{G} / \mathrm{T}$ & 0.3047 & -0.0755 & 0.8821 & h81.3 & CCTC & 0.0352 & 3.6130 & 1.4518 & 2.4886 & 0.0130 & \\
\hline \multirow[t]{5}{*}{81} & p62409-rs838911 & 125287111 & Intron 7 & $C / T$ & 0.4211 & -0.6245 & 0.1888 & h81.4 & $\mathrm{CCTT}$ & 0.2683 & -0.7498 & 0.6337 & -1.1832 & 0.2371 & \\
\hline & & & & & & & & h81.5 & CTGC & 0.0886 & 1.4787 & 0.9259 & 1.5970 & 0.1107 & \\
\hline & & & & & & & & h81.6 & CTGT & 0.1287 & -0.2477 & 0.7967 & -0.3109 & 0.7560 & \\
\hline & & & & & & & & h81.7 (rare) & $* * * *$ & 0.0017 & 4.9120 & 8.4190 & 0.5834 & 0.5598 & \\
\hline & & & & & & & & hap.base81 & TCGC & 0.2819 & NA & NA & NA & NA & \\
\hline 82 & p61872-rs838909 & 125287648 & Intron 7 & $C / T$ & 0.2199 & 0.9232 & 0.1056 & h82.1 & CGTT & 0.0214 & 0.3707 & 1.6055 & 0.2309 & 0.8175 & 0.0137 \\
\hline 82 & p62140-rs838910 & 125287380 & Intron 7 & $\mathrm{G} / \mathrm{T}$ & 0.3047 & -0.0755 & 0.8821 & h82.2 & СТCT & 0.0364 & 3.8641 & 1.3703 & 2.8199 & 0.0049 & \\
\hline
\end{tabular}


Table 7 Significant haplotype association (global $P<0.05$ ) of 136 SCARB1 genotyped variants with HDL-C and ApoA-I (Continued)

\begin{tabular}{|c|c|c|c|c|c|c|c|c|c|c|c|c|c|c|c|c|}
\hline 82 & p62409-rs838911 & 125287111 & Intron 7 & & $C / T$ & 0.4211 & -0.6245 & 0.1888 & h82.3 & $\mathrm{CTT}$ & 0.2692 & -0.2007 & 0.5674 & -0.3537 & 0.7237 & \\
\hline \multirow[t]{5}{*}{82} & p62615-rs7138386 & 125286905 & Intron 7 & & $\mathrm{~T} / \mathrm{C}$ & 0.1137 & -0.6495 & 0.3851 & h82.4 & TGCT & 0.0869 & 2.1488 & 0.8777 & 2.4481 & 0.0146 & \\
\hline & & & & & & & & & h82.5 & TGTT & 0.0179 & 3.0085 & 1.9599 & 1.5351 & 0.1252 & \\
\hline & & & & & & & & & h82.6 & TGTC & 0.1116 & -0.1961 & 0.7815 & -0.2510 & 0.8019 & \\
\hline & & & & & & & & & h82.7 (rare) & $* * * *$ & 0.0020 & -4.7635 & 9.0097 & -0.5287 & 0.5972 & \\
\hline & & & & & & & & & hap.base82 & CGCT & 0.4546 & NA & NA & NA & NA & \\
\hline 83 & p62140-rs838910 & 125287380 & Intron 7 & & $\mathrm{G} / \mathrm{T}$ & 0.3047 & -0.0755 & 0.8821 & h83.1 & GCTA & 0.0854 & 2.0624 & 0.8886 & 2.3211 & 0.0205 & 0.0187 \\
\hline 83 & p62409-rs838911 & 125287111 & Intron 7 & & $C / T$ & 0.4211 & -0.6245 & 0.1888 & h83.2 & GTTG & 0.0389 & 1.3667 & 1.2527 & 1.0910 & 0.2756 & \\
\hline 83 & p62615-rs7138386 & 125286905 & Intron 7 & & $\mathrm{~T} / \mathrm{C}$ & 0.1137 & -0.6495 & 0.3851 & h83.3 & GTCG & 0.1129 & -0.3143 & 0.7855 & -0.4002 & 0.6891 & \\
\hline \multirow[t]{4}{*}{83} & p63483-rs838912 & 125286037 & Intron 7 & & $\mathrm{G} / \mathrm{A}$ & 0.0867 & 1.8700 & 0.0234 & h83.4 & TCTG & 0.0368 & 3.8488 & 1.3757 & 2.7977 & 0.0053 & \\
\hline & & & & & & & & & h83.5 & TाTG & 0.2675 & -0.1681 & 0.5759 & -0.2918 & 0.7705 & \\
\hline & & & & & & & & & h83.6 (rare) & $* * * *$ & 0.0031 & -0.5696 & 5.5038 & -0.1035 & 0.9176 & \\
\hline & & & & & & & & & hap.base83 & GCTG & 0.4554 & NA & NA & NA & NA & \\
\hline 86 & p63483-rs838912 & 125286037 & Intron 7 & & $\mathrm{G} / \mathrm{A}$ & 0.0867 & 1.8700 & 0.0234 & h86.1 & ATCG & 0.0871 & 2.5431 & 0.8550 & 2.9743 & 0.0030 & 0.0290 \\
\hline 86 & p64772-rs5888 & 125284748 & Exon 8 & Ala350Ala & $C / T$ & 0.0961 & 2.0962 & 0.0080 & h86.2 & GCAG & 0.1457 & 0.3613 & 0.6957 & 0.5194 & 0.6037 & \\
\hline 86 & p64923-rs838915 & 125284597 & Intron 8 & & C/A & 0.1435 & -0.3684 & 0.5766 & h86.3 & GCCA & 0.2814 & 1.0972 & 0.5782 & 1.8976 & 0.0581 & \\
\hline \multirow[t]{2}{*}{86} & p65999-rs12819677 & 125283521 & Intron 8 & & $\mathrm{G} / \mathrm{A}$ & 0.2813 & 0.6769 & 0.2052 & h86.4 & GTCG & 0.0116 & 1.6563 & 2.1240 & 0.7798 & 0.4357 & \\
\hline & & & & & & & & & hap.base86 & GCCG & 0.4736 & NA & NA & NA & NA & \\
\hline 95 & p71867-rs7954022 & 125277653 & Intron 9 & & $C / T$ & 0.1323 & 0.8502 & 0.2241 & h95.1 & TACT & 0.1311 & 0.8202 & 0.7688 & 1.0669 & 0.2864 & 0.0131 \\
\hline 95 & p72197-rs838861 & 125277323 & Intron 9 & & $A / G$ & 0.3777 & -0.1507 & 0.7464 & h95.2 & CACC & 0.0507 & 0.3188 & 1.2809 & 0.2489 & 0.8035 & \\
\hline 95 & p72777-rs838862 & 125276743 & Intron 9 & & $C / T$ & 0.0887 & 0.7012 & 0.3938 & h95.3 & CGCT & 0.1846 & -0.7832 & 0.6960 & -1.1253 & 0.2608 & \\
\hline \multirow[t]{5}{*}{95} & p75766-rs838866 & 125273754 & Intron 9 & & $\mathrm{~T} / \mathrm{C}$ & 0.2116 & -0.0497 & 0.9306 & h95.4 & CGCC & 0.1022 & 0.7176 & 0.8581 & 0.8362 & 0.4033 & \\
\hline & & & & & & & & & h95.5 & CGTT & 0.0324 & 4.7525 & 1.5071 & 3.1534 & 0.0017 & \\
\hline & & & & & & & & & h95.6 & CGTC & 0.0582 & -1.3987 & 1.0854 & -1.2887 & 0.1979 & \\
\hline & & & & & & & & & h95.7 (rare) & $* * * *$ & 0.0009 & 18.2723 & NA & NA & NA & \\
\hline & & & & & & & & & hap.base95 & CACT & 0.4399 & NA & NA & NA & NA & \\
\hline 96 & p72197-rs838861 & 125277323 & Intron 9 & & $A / G$ & 0.3777 & -0.1507 & 0.7464 & h96.1 & $\mathrm{ACCT}$ & 0.0443 & 1.0796 & 1.2832 & 0.8413 & 0.4004 & 0.0484 \\
\hline 96 & p72777-rs838862 & 125276743 & Intron 9 & & $C / T$ & 0.0887 & 0.7012 & 0.3938 & h96.2 & GCTC & 0.1849 & -0.7979 & 0.6554 & -1.2176 & 0.2238 & \\
\hline 96 & p75766-rs838866 & 125273754 & Intron 9 & & $\mathrm{~T} / \mathrm{C}$ & 0.2116 & -0.0497 & 0.9306 & h96.3 & GCCT & 0.0727 & -0.3866 & 0.9478 & -0.4079 & 0.6835 & \\
\hline \multirow[t]{3}{*}{96} & p75778-rs7301120 & 125273742 & Intron 9 & & $C / T$ & 0.1135 & 0.3767 & 0.6174 & h96.4 & GCCC & 0.0282 & 1.9372 & 1.6107 & 1.2027 & 0.2295 & \\
\hline & & & & & & & & & h96.5 & GTTC & 0.0319 & 4.2363 & 1.4400 & 2.9419 & 0.0034 & \\
\hline & & & & & & & & & h96.6 & GTCC & 0.0595 & -1.3421 & 1.0101 & -1.3286 & 0.1844 & \\
\hline
\end{tabular}


Table 7 Significant haplotype association (global $P<0.05$ ) of 136 SCARB1 genotyped variants with HDL-C and ApoA-I (Continued)

\begin{tabular}{|c|c|c|c|c|c|c|c|c|c|c|c|c|c|c|c|}
\hline & & & & & & & & h96.7 (rare) & $* * * *$ & 0.0058 & -3.2342 & 3.8265 & -0.8452 & 0.3983 & \\
\hline & & & & & & & & hap.base96 & ACTC & 0.5728 & NA & NA & NA & NA & \\
\hline 97 & p72777-rs838862 & 125276743 & Intron 9 & $\mathrm{C} / \mathrm{T}$ & 0.0887 & 0.7012 & 0.3938 & h97.1 & СТСТ & 0.1997 & -1.0781 & 0.6237 & -1.7287 & 0.0843 & 0.0098 \\
\hline 97 & p75766-rs838866 & 125273754 & Intron 9 & $\mathrm{~T} / \mathrm{C}$ & 0.2116 & -0.0497 & 0.9306 & h97.2 & $\mathrm{CCT}$ & 0.1141 & 0.2005 & 0.7597 & 0.2639 & 0.7919 & \\
\hline 97 & p75778-rs7301120 & 125273742 & Intron 9 & $C / T$ & 0.1135 & 0.3767 & 0.6174 & h97.3 & СCCT & 0.0336 & 0.7963 & 1.3894 & 0.5731 & 0.5667 & \\
\hline \multirow[t]{4}{*}{97} & p76757-rs9919713 & 125272763 & Intron 9 & $\mathrm{~A} / \mathrm{T}$ & 0.4390 & -0.1860 & 0.6921 & h97.4 & $\pi \mathrm{TT}$ & 0.0301 & 4.3773 & 1.4494 & 3.0201 & 0.0026 & \\
\hline & & & & & & & & h97.5 & TCCT & 0.0588 & -1.4125 & 1.0117 & -1.3961 & 0.1631 & \\
\hline & & & & & & & & h97.6 (rare) & $* * * *$ & 0.0050 & -6.5869 & 3.6167 & -1.8213 & 0.0690 & \\
\hline & & & & & & & & hap.base97 & CTCA & 0.5587 & NA & NA & NA & NA & \\
\hline 109 & p78402-rs838898 & 125271118 & Intron 10 & $G / A$ & 0.0714 & -0.9806 & 0.2889 & h109.1 & AGCT & 0.0288 & -1.4134 & 1.6436 & -0.8600 & 0.3901 & 0.0195 \\
\hline 109 & p78430-rs838897 & 125271090 & Intron 10 & $C / G$ & 0.3830 & -0.1887 & 0.6887 & h109.2 & AGTT & 0.0451 & -1.5093 & 1.2496 & -1.2078 & 0.2275 & \\
\hline 109 & p78747-rs2293440 & 125270773 & Intron 11 & $\mathrm{~T} / \mathrm{C}$ & 0.4112 & -0.2984 & 0.5352 & h109.3 & GGCC & 0.0317 & 3.0784 & 1.3763 & 2.2366 & 0.0256 & \\
\hline \multirow[t]{4}{*}{109} & p78791-rs75289200 & 125270729 & Intron 11 & $\mathrm{~T} / \mathrm{C}$ & 0.0321 & 3.6568 & 0.0086 & h109.4 & GGCT & 0.1633 & -0.4126 & 0.6911 & -0.5971 & 0.5506 & \\
\hline & & & & & & & & h109.5 & GGTT & 0.1088 & -1.6537 & 0.8639 & -1.9142 & 0.0560 & \\
\hline & & & & & & & & h109.6 & GCCT & 0.1851 & -1.8104 & 0.7168 & -2.5256 & 0.0118 & \\
\hline & & & & & & & & hap.base109 & GCTT & 0.4363 & NA & NA & NA & NA & \\
\hline 110 & p78430-rs838897 & 125271090 & Intron 10 & $C / G$ & 0.3830 & -0.1887 & 0.6887 & h110.1 & GCCC & 0.0305 & 3.0357 & 1.4224 & 2.1342 & 0.0331 & 0.0012 \\
\hline 110 & p78747-rs2293440 & 125270773 & Intron 11 & $\mathrm{~T} / \mathrm{C}$ & 0.4112 & -0.2984 & 0.5352 & h110.2 & GCTG & 0.0189 & -3.0973 & 2.2833 & -1.3565 & 0.1753 & \\
\hline 110 & p78791-rs75289200 & 125270729 & Intron 11 & $\mathrm{~T} / \mathrm{C}$ & 0.0321 & 3.6568 & 0.0086 & h110.3 & GCTC & 0.1696 & -0.0290 & 0.6830 & -0.0424 & 0.9662 & \\
\hline \multirow[t]{7}{*}{110} & p79721-rs838896 & 125269799 & Intron 11 & $\mathrm{G} / \mathrm{C}$ & 0.3104 & 1.1147 & 0.0278 & h110.4 & GTTG & 0.1400 & -2.3158 & 0.7741 & -2.9914 & 0.0029 & \\
\hline & & & & & & & & h110.5 & GTC & 0.0189 & 1.3536 & 2.3385 & 0.5788 & 0.5629 & \\
\hline & & & & & & & & h110.6 & CCTG & 0.1379 & -2.4014 & 0.7888 & -3.0443 & 0.0024 & \\
\hline & & & & & & & & h110.7 & ССTC & 0.0514 & -0.8677 & 1.2628 & -0.6871 & 0.4922 & \\
\hline & & & & & & & & h110.8 & СTTC & 0.0398 & -0.1892 & 1.4963 & -0.1264 & 0.8994 & \\
\hline & & & & & & & & h110.9 (rare) & $* * * *$ & 0.0012 & 7.8235 & 8.0313 & 0.9741 & 0.3303 & \\
\hline & & & & & & & & hap.base110 & CTTG & 0.3918 & NA & NA & NA & NA & \\
\hline 111 & p78747-rs2293440 & 125270773 & Intron 11 & $\mathrm{~T} / \mathrm{C}$ & 0.4112 & -0.2984 & 0.5352 & h111.1 & CCCG & 0.0305 & 3.5704 & 1.4077 & 2.5364 & 0.0114 & 0.0038 \\
\hline 111 & p78791-rs75289200 & 125270729 & Intron 11 & $\mathrm{~T} / \mathrm{C}$ & 0.0321 & 3.6568 & 0.0086 & h111.2 & CTGC & 0.1514 & -2.1697 & 0.7058 & -3.0742 & 0.0022 & \\
\hline 111 & p79721-rs838896 & 125269799 & Intron 11 & $\mathrm{G} / \mathrm{C}$ & 0.3104 & 1.1147 & 0.0278 & h111.3 & CTCG & 0.2233 & 0.3086 & 0.5985 & 0.5157 & 0.6062 & \\
\hline \multirow[t]{3}{*}{111} & p79828-rs838895 & 125269692 & Intron 11 & $C / G$ & 0.3171 & 1.2206 & 0.0164 & h111.4 & TTGG & 0.0173 & 1.0502 & 1.9388 & 0.5417 & 0.5882 & \\
\hline & & & & & & & & h111.5 & $\pi G C$ & 0.0431 & 0.3464 & 1.3140 & 0.2637 & 0.7921 & \\
\hline & & & & & & & & h111.6 & $\pi \mathrm{T} C \mathrm{C}$ & 0.0150 & 0.6429 & 1.9745 & 0.3256 & 0.7448 & \\
\hline
\end{tabular}


Table 7 Significant haplotype association (global $P<0.05)$ of 136 SCARB1 genotyped variants with HDL-C and ApoA-I (Continued)

\begin{tabular}{|c|c|c|c|c|c|c|c|c|c|c|c|c|c|c|c|}
\hline & & & & & & & & h111.7 (rare) & $* * * *$ & 0.0047 & 3.8853 & 4.0634 & 0.9562 & 0.3393 & \\
\hline & & & & & & & & hap.base111 & TTGC & 0.5147 & NA & NA & NA & NA & \\
\hline 112 & p78791-rs75289200 & 125270729 & Intron 11 & $\mathrm{~T} / \mathrm{C}$ & 0.0321 & 3.6568 & 0.0086 & h112.1 & CCGA & 0.0309 & 3.7315 & 1.3947 & 2.6755 & 0.0076 & 0.0412 \\
\hline 112 & p79721-rs838896 & 125269799 & Intron 11 & $\mathrm{G} / \mathrm{C}$ & 0.3104 & 1.1147 & 0.0278 & h112.2 & TGGA & 0.0179 & 1.8646 & 1.8467 & 1.0097 & 0.3130 & \\
\hline 112 & p79828-rs838895 & 125269692 & Intron 11 & $C / G$ & 0.3171 & 1.2206 & 0.0164 & h112.3 & TGCA & 0.0109 & -3.3720 & 2.3180 & -1.4547 & 0.1462 & \\
\hline \multirow[t]{4}{*}{112} & p80045-rs838893 & 125269475 & Intron 11 & $\mathrm{G} / \mathrm{A}$ & 0.3244 & 0.8859 & 0.0774 & h112.4 & TCGA & 0.2661 & 0.7087 & 0.5428 & 1.3056 & 0.1921 & \\
\hline & & & & & & & & h112.5 & TCCG & 0.0144 & 1.0316 & 2.0147 & 0.5120 & 0.6088 & \\
\hline & & & & & & & & h112.6 (rare) & $* * * *$ & 0.0068 & 2.8715 & 3.2105 & 0.8944 & 0.3714 & \\
\hline & & & & & & & & hap.base112 & TGCG & 0.6530 & NA & NA & NA & NA & \\
\hline 123 & p83884-rs701106 & 125265636 & Intron 12 & $C / T$ & 0.2597 & 1.2967 & 0.0156 & h123.1 & TCCT & 0.0235 & -1.7638 & 1.7393 & -1.0141 & 0.3109 & 0.0468 \\
\hline 123 & p86245-rs188375019 & 125263275 & Intron 12 & $C / T$ & 0.0341 & 1.8399 & 0.1674 & h123.2 & TCCG & 0.2351 & 1.8726 & 0.6006 & 3.1179 & 0.0019 & \\
\hline 123 & p86276-rs747155 & 125263244 & Intron 12 & $C / T$ & 0.1495 & -0.2164 & 0.7433 & h123.3 & CCTG & 0.1485 & 0.3912 & 0.6981 & 0.5604 & 0.5754 & \\
\hline \multirow[t]{4}{*}{123} & p86316-rs701104 & 125263204 & Intron 12 & $\mathrm{G} / \mathrm{T}$ & 0.0487 & -0.6627 & 0.5579 & h123.4 & ССCT & 0.0238 & 1.6476 & 1.7546 & 0.9390 & 0.3480 & \\
\hline & & & & & & & & h123.5 & CTCG & 0.0328 & 2.3144 & 1.3655 & 1.6949 & 0.0905 & \\
\hline & & & & & & & & h123.6 (rare) & $* * * *$ & 0.0024 & 1.2704 & 8.8153 & 0.1441 & 0.8855 & \\
\hline & & & & & & & & hap.base123 & CCCG & 0.5340 & NA & NA & NA & NA & \\
\hline
\end{tabular}

ApoA-I apolipoprotein A-I, Coef coefficient, del/D deletion, HDL-C high-density lipoprotein cholesterol, MAF minor allele frequency, NA not analyzed, SE standard error, SNP single nucleotide polymorphism, UTR untranslated region, $W$ wild type allele for deletion on RefSeq

All alleles on the reverse strand. HDL-C and ApoA-I variables were in $\mathrm{mg} / \mathrm{dL}$ and Box-Cox transformed

Results were adjusted for covariates: sex, age, waist, current smoking (yes/no), and minutes of daily walking or biking to work (jobmin) for HDL-C; sex and age for ApoA-I

SNP 1-SNP 4 for each window are shown as "SNP name-SNP ID/Chromosome 12 Position (for novel variants)". All 10 novel variants identified in this study have been submitted to dbSNP database (batch ID:

SCARB1_AB): http://www.ncbi.nlm.nih.gov/SNP/snp_viewTable.cgi?handle=KAMBOH.

Nominally significant $P$-values $(P<0.05)$ for SNPs with MAF $\geq 5 \%$ in single-site analysis are shown in bold

Haplotype sequences corresponding to SNP 1-SNP 4 in the $5^{\prime}$ to $3^{\prime}$ direction, respectively

Haplotype association results for all haplotype windows are shown in Additional file 16: Table S11, see haplotype association plots in Fig. 3

a, CRefSeq of SCARB1: hg19, NM_005505 (CHIP Bioinformatics)

${ }^{b}$ dbSNP build 139: GRCh37.p10 
Table 8 Significantly associated haplotype regions (global $P<0.05$ ) with HDL-C and ApoA-I

\begin{tabular}{|c|c|c|c|c|c|c|c|c|c|}
\hline \multirow[t]{3}{*}{ Region \# } & \multirow[t]{3}{*}{ Trait } & \multicolumn{8}{|c|}{ Consecutive Significantly Associated Haplotype Windows (global $P<0.05$ ) } \\
\hline & & \multirow{2}{*}{$\begin{array}{l}\text { Haplotype } \\
\text { Windows } \\
\#\end{array}$} & \multicolumn{2}{|c|}{$\frac{\text { Chr12 Position }^{a}}{\text { (Location) }}$} & \multicolumn{2}{|c|}{ The Composited Variants in the Region, $5^{\prime}$ to $3^{\prime}$ Direction } & \multicolumn{3}{|c|}{ Most Relevant Haplotype } \\
\hline & & & Start $\left(5^{\prime}\right)$ & End $\left(3^{\prime}\right)$ & SNP Name ${ }^{b}-S N P$ IDc/Chr12 Position ${ }^{a}$ & Major/Minor Alleles & Haplotype \# & Sequence & $\beta$ (Min-Max) \\
\hline \multirow[t]{8}{*}{1} & $\mathrm{HDL}-\mathrm{C}$ & 44 & 125300551 & 125299542 & p48969-rs2343394 & $\mathrm{C} / \mathrm{T}$ & h44.3 & CCWGCGG & $0.4910-1.0491$ \\
\hline & & 45 & (intron 2) & (exon 3) & p49537-rs7305310 & $\mathrm{C} / \mathrm{T}$ & h45.2 & & \\
\hline & & 46 & & & p49570delC-rs 145376237 & W/D & h46.2 & & \\
\hline & & 47 & & & p49690-rs4765615 & $\mathrm{G} / \mathrm{A}$ & hap.base47 & & \\
\hline & & & & & p49759-rs146272788 & $\mathrm{C} / \mathrm{T}$ & hap.base44 & CCWACGG & -0.4701 \\
\hline & & & & & p49978-rs5891 (p.Val135Ile) & $\mathrm{G} / \mathrm{A}$ & hap.base45 & & \\
\hline & & & & & p50024-rs368880622 & $\mathrm{G} / \mathrm{T}$ & hap.base46 & & \\
\hline & & & & & & & h47.1 & & \\
\hline \multirow[t]{6}{*}{2} & ApoA-1 & 47 & 125299830 & 125299369 & p49690-rs4765615 & $\mathrm{G} / \mathrm{A}$ & h47.1 & ACGGTT & $(-0.8907)-3.3792$ \\
\hline & & 48 & (intron 2) & (intron 3) & p49759-rs146272788 & $\mathrm{C} / \mathrm{T}$ & h48.1 & & \\
\hline & & 49 & & & p49978-rs5891 (p.Val135Ile) & $\mathrm{G} / \mathrm{A}$ & h49.1 & & \\
\hline & & & & & p50024-rs368880622 & $\mathrm{G} / \mathrm{T}$ & & & \\
\hline & & & & & p50118-rs58710319 & $\mathrm{C} / \mathrm{T}$ & & & \\
\hline & & & & & p50151-rs2278986 & $\mathrm{T} / \mathrm{C}$ & & & \\
\hline \multirow[t]{6}{*}{3} & ApoA-I & 70 & 125294893 & 125292516 & p54627-chr12_125294893 & $\mathrm{G} / \mathrm{C}$ & h70.2 & GCGTAG & $2.0304-2.1103$ \\
\hline & & 71 & (intron 5) & (intron 6) & p54856-chr12_125294664 ${ }^{d}$ & $\mathrm{C} / \mathrm{T}$ & h71.3 & & \\
\hline & & 72 & & & p55923-rs838900 & $\mathrm{G} / \mathrm{A}$ & h72.3 & & \\
\hline & & & & & p55963-rs7134858 & $\mathrm{C} / \mathrm{T}$ & & & \\
\hline & & & & & p56845-rs838902 & $\mathrm{A} / \mathrm{G}$ & & & \\
\hline & & & & & p57004-rs187562853 & $\mathrm{G} / \mathrm{A}$ & & & \\
\hline \multirow[t]{8}{*}{4} & ApoA-I & 78 & 125291928 & 125286037 & p57592-rs838903 & $\mathrm{G} / \mathrm{A}$ & h78.5 & GTTCGCTG & $4.7307-5.5715$ \\
\hline & & 79 & (intron 7) & (intron 7) & p58514-rs838905 & $\mathrm{T} / \mathrm{C}$ & h79.6 & & \\
\hline & & 80 & & & p58664-rs865716 & $A / T$ & h80.6 & & \\
\hline & & 81 & & & p60255-rs3782287 & $\mathrm{C} / \mathrm{T}$ & hap.base81 & & \\
\hline & & 82 & & & p61872-rs838909 & $\mathrm{C} / \mathrm{T}$ & hap.base82 & & \\
\hline & & 83 & & & p62140-rs838910 & $\mathrm{G} / \mathrm{T}$ & hap.base83 & & \\
\hline & & & & & p62409-rs838911 & $\mathrm{C} / \mathrm{T}$ & h78.2 & GTACCTCTG & $0.6384-3.8641$ \\
\hline & & & & & p62615-rs7138386 & $T / C$ & h79.2 & & \\
\hline
\end{tabular}


Table 8 Significantly associated haplotype regions (global $P<0.05$ ) with $\mathrm{HDL}-\mathrm{C}$ and ApoA-I (Continued)

\begin{tabular}{|c|c|c|c|c|c|c|c|c|c|}
\hline & & & & & p63483-rs838912 & $\mathrm{G} / \mathrm{A}$ & hap.base80 & & \\
\hline & & & & & & & h81.3 & & \\
\hline & & & & & & & h82.2 & & \\
\hline & & & & & & & h83.4 & & \\
\hline \multirow[t]{6}{*}{5} & ApoA-1 & 95 & 125277653 & 125272763 & p71867-rs7954022 & $\mathrm{C} / \mathrm{T}$ & h95.5 & CGTTCT & $4.2363-4.7525$ \\
\hline & & 96 & (intron 9) & (intron 9) & p72197-rs838861 & $\mathrm{A} / \mathrm{G}$ & h96.5 & & \\
\hline & & 97 & & & p72777-rs838862 & $\mathrm{C} / \mathrm{T}$ & h97.4 & & \\
\hline & & & & & p75766-rs838866 & $\mathrm{T} / \mathrm{C}$ & & & \\
\hline & & & & & p75778-rs7301120 & $\mathrm{C} / \mathrm{T}$ & & & \\
\hline & & & & & p76757-rs9919713 & $A / T$ & & & \\
\hline \multirow[t]{7}{*}{$6^{*}$} & ApoA-I & 109 & 125271118 & 125269475 & p78402-rs838898 & $\mathrm{G} / \mathrm{A}$ & h109.6 & GCCTGCA & $(-3.3720)-(-1.8104)$ \\
\hline & & 110 & (intron 10) & (intron 11) & p78430-rs838897 & $C / G$ & h110.6 & & \\
\hline & & 111 & & & p78747-rs2293440 & $\mathrm{T} / \mathrm{C}$ & h111.2 & & \\
\hline & & 112 & & & p78791-rs75289200 & $\mathrm{T} / \mathrm{C}$ & h112.3 & & \\
\hline & & & & & p79721-rs838896 & $\mathrm{G} / \mathrm{C}$ & & & \\
\hline & & & & & p79828-rs838895 & $C / G$ & & & \\
\hline & & & & & p80045-rs838893 & $\mathrm{G} / \mathrm{A}$ & & & \\
\hline \multirow[t]{7}{*}{$7^{*}$} & $\mathrm{HDL}-\mathrm{C}$ & 111 & 125270773 & 125267501 & p78747-rs2293440 & $\mathrm{T} / \mathrm{C}$ & h111.4 & TTGGAGC & $0.3755-2.3949$ \\
\hline & & 112 & (intron 11) & (intron 11) & p78791-rs75289200 & $\mathrm{T} / \mathrm{C}$ & h112.2 & & \\
\hline & & 113 & & & p79721-rs838896 & $\mathrm{G} / \mathrm{C}$ & h113.1 & & \\
\hline & & 114 & & & p79828-rs838895 & $C / G$ & h114.1 & & \\
\hline & & & & & p80045-rs838893 & $\mathrm{G} / \mathrm{A}$ & & & \\
\hline & & & & & p81863-rs185445624 & $\mathrm{G} / \mathrm{A}$ & & & \\
\hline & & & & & p82019-rs838890 & $\mathrm{C} / \mathrm{T}$ & & & \\
\hline \multirow[t]{5}{*}{8} & $\mathrm{HDL}-\mathrm{C}$ & 117 & 125267501 & 125267086 & p82019-rs838890 & $\mathrm{C} / \mathrm{T}$ & h117.2 & TCGGC & $(-1.0134)-(-0.9657)$ \\
\hline & & 118 & (intron 11) & (intron 12) & p82264-rs141545424 (p.Gly501Gly) & $\mathrm{C} / \mathrm{A}$ & h118.2 & & \\
\hline & & & & & p82340-rs77483223 & $\mathrm{G} / \mathrm{A}$ & & & \\
\hline & & & & & p82369-rs75446635 & $\mathrm{G} / \mathrm{A}$ & & & \\
\hline & & & & & p82434-rs838889 & $\mathrm{T} / \mathrm{C}$ & & & \\
\hline
\end{tabular}


Table 8 Significantly associated haplotype regions (global $P<0.05$ ) with HDL-C and ApoA-I (Continued)

\begin{tabular}{|c|c|c|c|c|c|c|c|c|c|}
\hline \multirow[t]{6}{*}{9} & $\mathrm{HDL}-\mathrm{C}$ & 123 & 125265636 & 125262553 & p83884-rs701106 & $\mathrm{C} / \mathrm{T}$ & h123.4 & CCCTGA & $(-1.180)-(-0.2329)$ \\
\hline & & 124 & (intron 12) & (exon 13-3' UTR) & p86245-rs188375019 & $\mathrm{C} / \mathrm{T}$ & h124.2 & & \\
\hline & & 125 & & & p86276-rs747155 & $\mathrm{C} / \mathrm{T}$ & h125.2 & & \\
\hline & & & & & p86316-rs701104 & $\mathrm{G} / \mathrm{T}$ & & & \\
\hline & & & & & p86481-rs701103 (p.Gly499Arg, isoform 2) & $\mathrm{G} / \mathrm{A}$ & & & \\
\hline & & & & & p86967-rs187492239 & $\mathrm{A} / \mathrm{G}$ & & & \\
\hline
\end{tabular}

p86967-rs187492239

ApoA-I apolipoprotein A-I, del/D deletion, HDL-C high-density lipoprotein cholesterol, SNP single nucleotide polymorphism, UTR untranslated region, $W$ wild type allele for deletion on the RefSea All alleles on the reverse strand. HDL-C and ApoA-I variables were in $\mathrm{mg} / \mathrm{dL}$ and Box-Cox transformed

Results were adjusted for covariates: sex, age, waist, current smoking (yes/no), and minutes of daily walking or biking to work (jobmin) for HDL-C; sex and age for ApoA-1

All nine haplotype regions are shown in Fig. 3

Detailed single-site associations are shown in Additional file 14: Table S9 and Additional file 15: Table S10

Detailed haplotype associations are shown in Table 7 and Additional file 16: Table S11

Regions with asterisk $\left(^{*}\right)$ indicate regions that included the haplotype window exhibiting the most significant association signal (the smallest global $P$ ) for the associated trait

For each region, the most significant associated haplotype window is shown in bold

SNPs with significant evidence of association with the same trait in both single-site and haplotype analyses (single-site $P<0.05$ and global $P<0.05$ ) are shown in bold

SNPs with significant evidence of association with different trait in single-site and haplotype analyses (single-site $P<0.05$ and global $P<0.05$ ) are shown in italic bold

a, befSeq of SCARB1: hg19, NM_005505 (CHIP Bioinformatics)

cdbSNP build 139: GRCh37.p10

${ }^{d}$ Rare variants of interest with potential effects on lipid traits; see details in Table 6 


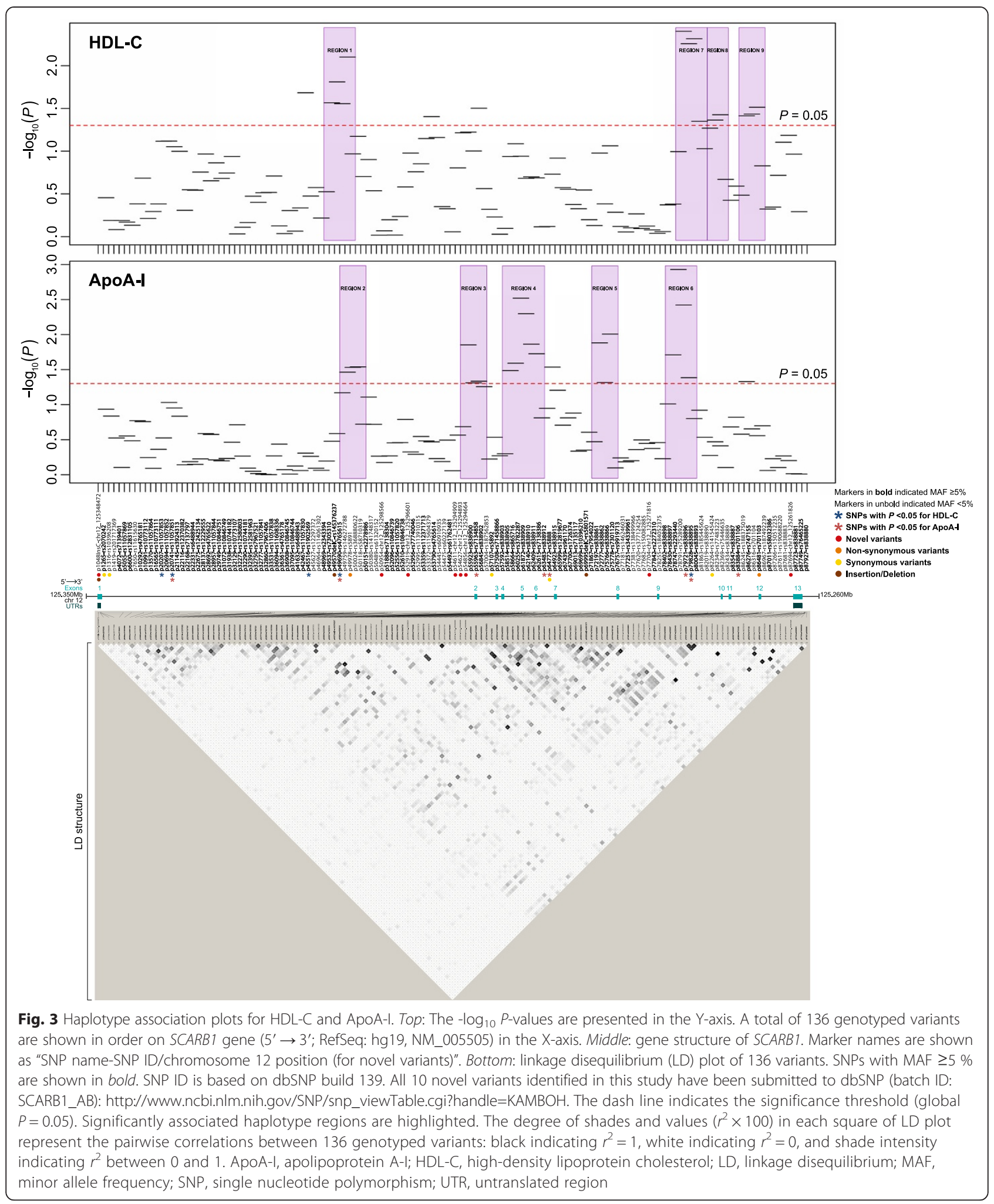

788 African Blacks. Additional 69 tagSNPs from the HapMap-YRI data along with two previously reported lipid-associated SCARB1 variants were also genotyped in the total sample. Of 149 genotyped SCARB1 variants,
137 that passed QC were examined for association with major lipid traits (Table 2). The initial gene-based analyses revealed a nominal association with HDL-C $(P=0.0421)$ as well as a trend for association with ApoA-I $(P=0.1016$; 
Table 9 Results for 7 SCARB1 lipid-associated variants in US Non-Hispanic Whites (previous study ${ }^{a}$ ) and in African Blacks (this study)

\begin{tabular}{|c|c|c|c|c|c|c|c|c|c|c|c|c|}
\hline \multirow{2}{*}{$\begin{array}{l}\text { SNP } \\
\text { Name }^{b}\end{array}$} & \multirow[t]{2}{*}{ SNP ID } & \multirow{2}{*}{$\begin{array}{l}\text { Chr12 } \\
\text { Positiond }^{d}\end{array}$} & \multirow[t]{2}{*}{ Location } & \multirow{2}{*}{$\begin{array}{l}\text { RegDB } \\
\text { Score }\end{array}$} & \multirow[t]{2}{*}{ Alleles } & \multicolumn{3}{|c|}{ US Non-Hispanic Whites ${ }^{\mathrm{a}}(n=623)$} & \multicolumn{4}{|c|}{ African Blacks $(n=788)$} \\
\hline & & & & & & MA, MAF & $\begin{array}{l}\beta \\
(\mathrm{SE})\end{array}$ & $P$ & $\begin{array}{l}\text { MA, } \\
\text { MAF }\end{array}$ & $\begin{array}{l}\beta \\
(\mathrm{SE})\end{array}$ & $P$ & $\begin{array}{l}\text { Other Assoc } \\
\text { Trait(s) }^{f}\end{array}$ \\
\hline \multicolumn{13}{|l|}{$\mathrm{HDL}-\mathrm{C}$} \\
\hline \multirow[t]{2}{*}{ p28957 } & rs11057844 & 125320563 & Intron 1 & 5 & $\mathrm{G} / \mathrm{A}$ & A, 0.1839 & -0.0395 & 0.0035 & A, 0.2362 & 0.3671 & 0.1075 & \\
\hline & & & & & & & $(0.0135)$ & & & $(0.2278)$ & & \\
\hline \multirow[t]{2}{*}{ p83884 } & rs701106 & 125265636 & Intron 12 & 5 & $C / T$ & T, 0.1527 & 0.0394 & 0.0066 & $T, 0.2597$ & 0.2471 & 0.2601 & ApoA-I \\
\hline & & & & & & & $(0.0144)$ & & & $(0.2192)$ & & \\
\hline \multirow[t]{2}{*}{ p87927 } & rs838880 & 125261593 & 3' flanking & 5 & $\mathrm{G} / \mathrm{A}$ & G, 0.3237 & 0.0257 & 0.0250 & A, 0.2414 & 0.0198 & 0.9314 & \\
\hline & & & & & & & $(0.0114)$ & & & $(0.2302)$ & & \\
\hline \multicolumn{13}{|l|}{ ApoB } \\
\hline \multirow[t]{2}{*}{ p48969 } & rs2343394 & 125300551 & Intron 2 & 5 & $\mathrm{C} / \mathrm{T}$ & T, 0.2850 & 1.2544 & 0.0082 & $T, 0.1898$ & 0.0383 & 0.9544 & \\
\hline & & & & & & & $(0.4721)$ & & & $(0.6696)$ & & \\
\hline \multirow[t]{2}{*}{ p49690 } & rs4765615 & 125299830 & Intron 2 & 5 & $\mathrm{G} / \mathrm{A}$ & G, 0.4497 & 1.2493 & 0.0059 & A, 0.4426 & 0.7771 & 0.1338 & HDL-C, ApoA-I \\
\hline & & & & & & & $(0.4518)$ & & & $(0.5178)$ & & \\
\hline \multirow[t]{2}{*}{ p50151 } & rs2278986 & 125299369 & Intron 3 & 5 & T/C & C, 0.2890 & 1.1926 & 0.0122 & C, 0.1933 & 0.1308 & 0.8434 & \\
\hline & & & & & & & $(0.4735)$ & & & (0.6619) & & \\
\hline \multirow[t]{2}{*}{ p52556 } & rs11057820 & 125296964 & Intron 4 & 5 & $\mathrm{G} / \mathrm{A}$ & $\mathrm{G}, 0.4871$ & 0.8700 & 0.0436 & $A, 0.1000$ & 1.8661 & 0.0292 & \\
\hline & & & & & & & $(0.4300)$ & & & $(0.8542)$ & & \\
\hline
\end{tabular}

$A p o B$ apolipoprotein B, HDL-C high-density lipoprotein cholesterol, MA minor allele, MAF minor allele frequency, RegDB RegulomeDB, SE standard error, SNP single nucleotide polymorphism

All alleles on the reverse strand

HDL-C and ApoB values for US Non-Hispanic Whites were in mg/dL, Box-Cox transformed, and adjusted for covariates: sex, age, body mass index, and smoking (past/current/never) for HDL-C; age and smoking

HDL-C and ApoB values for African Blacks were in mg/dL, Box-Cox transformed, and adjusted for covariates: sex, age, waist, current smoking (yes/no), and daily walking or biking to work (jobmin) for HDL-C; body mass index and staff status for ApoB

Nominally significant $P$-values $(P<0.05)$ are shown in bold

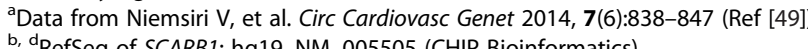

b, dRefSeq of SCARB1: hg19, NM_005505 (CHIP Bioinformatics)

'dbSNP version 139: GRCh37.p10

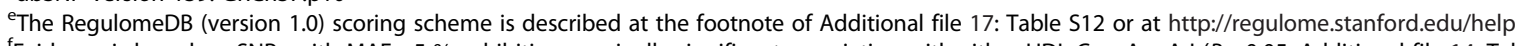

${ }^{\mathrm{f}}$ Evidence is based on SNPs with MAF $\geq 5 \%$ exhibiting nominally significant association with either HDL-C or ApoA-I $(P<0.05$; Additional file 14: Table S9 and Additional file 15: Table S10) in single-site association

results in the current study 
Table 10 Significant lipid-associated regions (global $P<0.05$ ) that were observed in US Non-Hispanic Whites (previous study ${ }^{\mathrm{a}}$ ) and African Blacks (this study)

\begin{tabular}{|c|c|c|c|c|c|c|c|c|c|c|c|c|}
\hline \multirow{3}{*}{$\begin{array}{l}\text { Region } \\
\#\end{array}$} & \multicolumn{6}{|c|}{ Consecutive Haplotype Windows in 623 US Non-Hispanic Whites ${ }^{a}$} & \multicolumn{6}{|c|}{ Consecutive Haplotype Windows in 788 African Blacks } \\
\hline & \multirow[t]{2}{*}{ Trait } & \multicolumn{2}{|c|}{ Chr12 Position ${ }^{\mathrm{b}}$ (Location) } & \multirow[t]{2}{*}{$\begin{array}{l}\text { Length } \\
\text { (bp) }\end{array}$} & \multicolumn{2}{|c|}{ The Composited Variants, 5' to $3^{\prime}$ Direction } & \multirow[t]{2}{*}{ Trait } & \multicolumn{2}{|c|}{ Chr12 Position ${ }^{\mathrm{b}}$ (Location) } & \multirow[t]{2}{*}{$\begin{array}{l}\text { Length } \\
\text { (bp) }\end{array}$} & \multicolumn{2}{|c|}{$\begin{array}{l}\text { The Composited Variants, } 5^{\prime} \text { to } 3^{\prime} \\
\text { Direction }\end{array}$} \\
\hline & & Start (5') & End $\left(3^{\prime}\right)$ & & SNP Name ${ }^{c-} S N P I^{d}$ & $\begin{array}{l}\text { Major/Minor } \\
\text { Alleles }\end{array}$ & & Start (5') & End $\left(3^{\prime}\right)$ & & SNP Name ${ }^{c}-S N P I^{d}$ & $\begin{array}{l}\text { Major/Minor } \\
\text { Alleles }\end{array}$ \\
\hline \multirow[t]{16}{*}{1} & ApoB & 125300551 & 125299369 & 1183 & p48969-rs2343394 & $\mathrm{C} / \mathrm{T}$ & HDL-C & 125300551 & 125299496 & 1056 & p48969-rs2343394 & $\mathrm{C} / \mathrm{T}$ \\
\hline & & (intron 2) & (intron 3) & & p49518-rs144194221 & $\mathrm{G} / \mathrm{A}$ & & (intron 2) & (intron 3) & & p49537-rs7305310 & $\mathrm{C} / \mathrm{T}$ \\
\hline & & & & & p49690-rs4765615 & $A / G$ & & & & & $\begin{array}{l}\text { p49570delC- } \\
\text { rs145376237 }\end{array}$ & W/D \\
\hline & & & & & p49978-rs5891 & $\mathrm{G} / \mathrm{A}$ & & & & & p49690-rs4765615 & $\mathrm{G} / \mathrm{A}$ \\
\hline & & & & & (p.Val135Ile) & & & & & & & \\
\hline & & & & & p50151-rs2278986 & T/C & & & & & p49759-rs146272788 & $C / T$ \\
\hline & & & & & & & & & & & p49978-rs5891 & $\mathrm{G} / \mathrm{A}$ \\
\hline & & & & & & & & & & & (p.Val135Ile) & \\
\hline & & & & & & & & & & & p50024-rs368880622 & $\mathrm{G} / \mathrm{T}$ \\
\hline & & & & & & & ApoA-I & 125299830 & 125299369 & 462 & p49690-rs4765615 & $\mathrm{G} / \mathrm{A}$ \\
\hline & & & & & & & & (intron 2) & (intron 3) & & p49759-rs146272788 & $\mathrm{C} / \mathrm{T}$ \\
\hline & & & & & & & & & & & p49978-rs5891 & $\mathrm{G} / \mathrm{A}$ \\
\hline & & & & & & & & & & & (p.Val135lle) & \\
\hline & & & & & & & & & & & p50024-rs368880622 & $\mathrm{G} / \mathrm{T}$ \\
\hline & & & & & & & & & & & p50118-rs58710319 & $C / T$ \\
\hline & & & & & & & & & & & p50151-rs2278986 & $\mathrm{T} / \mathrm{C}$ \\
\hline \multirow[t]{11}{*}{ II } & HDL-C & 125269692 & 125262516 & 7177 & p79828-rs838895 & $C / G$ & HDL-C & 125269692 & 125267501 & 2192 & p79828-rs838895 & $C / G$ \\
\hline & & (intron 11) & $\begin{array}{l}\text { (exon 13- 3' } \\
\text { UTR) }\end{array}$ & & p80045-rs838893 & $\mathrm{G} / \mathrm{A}$ & & (intron 11) & (intron 11) & & p80045-rs838893 & $\mathrm{G} / \mathrm{A}$ \\
\hline & & & & & p83088-rs797729 & $A / G$ & & & & & p81863-rs185445624 & $\mathrm{G} / \mathrm{A}$ \\
\hline & & & & & p83884-rs701106 & $\mathrm{C} / \mathrm{T}$ & & & & & p82019-rs838890 & $C / T$ \\
\hline & & & & & p86436-rs10396214 & $\mathrm{C} / \mathrm{T}$ & HDL-C & 125267501 & 125267086 & 416 & p82019-rs838890 & $C / T$ \\
\hline & & & & & (p.Arg484Trp, isoform 2 & & & & & & & \\
\hline & & & & & p87004-rs184715678 & $C / A$ & & (intron 11) & (intron 12) & & p82264-rs141545424 & $\mathrm{C} / \mathrm{A}$ \\
\hline & & & & & & & & & & & (p.Gly501Gly) & \\
\hline & & & & & & & & & & & p82340-rs77483223 & $\mathrm{G} / \mathrm{A}$ \\
\hline & & & & & & & & & & & p82369-rs75446635 & $\mathrm{G} / \mathrm{A}$ \\
\hline & & & & & & & & & & & p82434-rs838889 & $\mathrm{T} / \mathrm{C}$ \\
\hline
\end{tabular}


Table 10 Significant lipid-associated regions (global $P<0.05)$ that were observed in US Non-Hispanic Whites (previous study ${ }^{\mathrm{a}}$ ) and African Blacks (this study) (Continued)

HDL-C 125265636125262553

3084

p83884-rs701106

(intron 12) (exon 13- 3'

p86245-rs188375019

$\mathrm{C} / \mathrm{T}$

p86276-rs747155

p86316-rs701104

p86481-rs701103

(p.Gly499Arg, isoform 2)

p86967-rs187492239

$A / G$

ApoA-I apolipoprotein A-I, ApoB apolipoprotein B, del/D deletion, HDL-C high-density lipoprotein cholesterol, SNP single nucleotide polymorphism, UTR untranslated region, W wild type allele for deletion on RefSeq

All alleles on the reverse strand
Results for a US Non-Hispanic White sample were Box-Cox transformed, and adjusted for covariates: sex, age, body mass index, and smoking (past/current/never) for HDL-C; age and smoking for ApoB

Results for an African Black sample were Box-Cox transformed, and adjusted for covariates: sex, age, waist, current smoking (yes/no), and minutes of walking or biking to work each day (jobmin) for HDL-C; sex and age for ApoA-I

SNPs with significant evidence with the same trait in both single-site and haplotype associations (single-site $P$ and global $P<0.05$ ) observed in each population are shown in bold

SNPs with significant evidence with the different trait in single-site and haplotype associations (single-site $P$ and global $P<0.05$ ) in each population are shown in italic bold

SNPs with significant evidence with the different trait in single-site and haploty
${ }^{2}$ Data from Niemsiri V, et al. Circ Cardiovasc Genet 2014, 7(6):838-847 (Ref [49])

b, cRefSeq of SCARB1: hg19, NM_005505 (CHIP Bioinformatics)

ddbSNP version 139: GRCh37.p10 


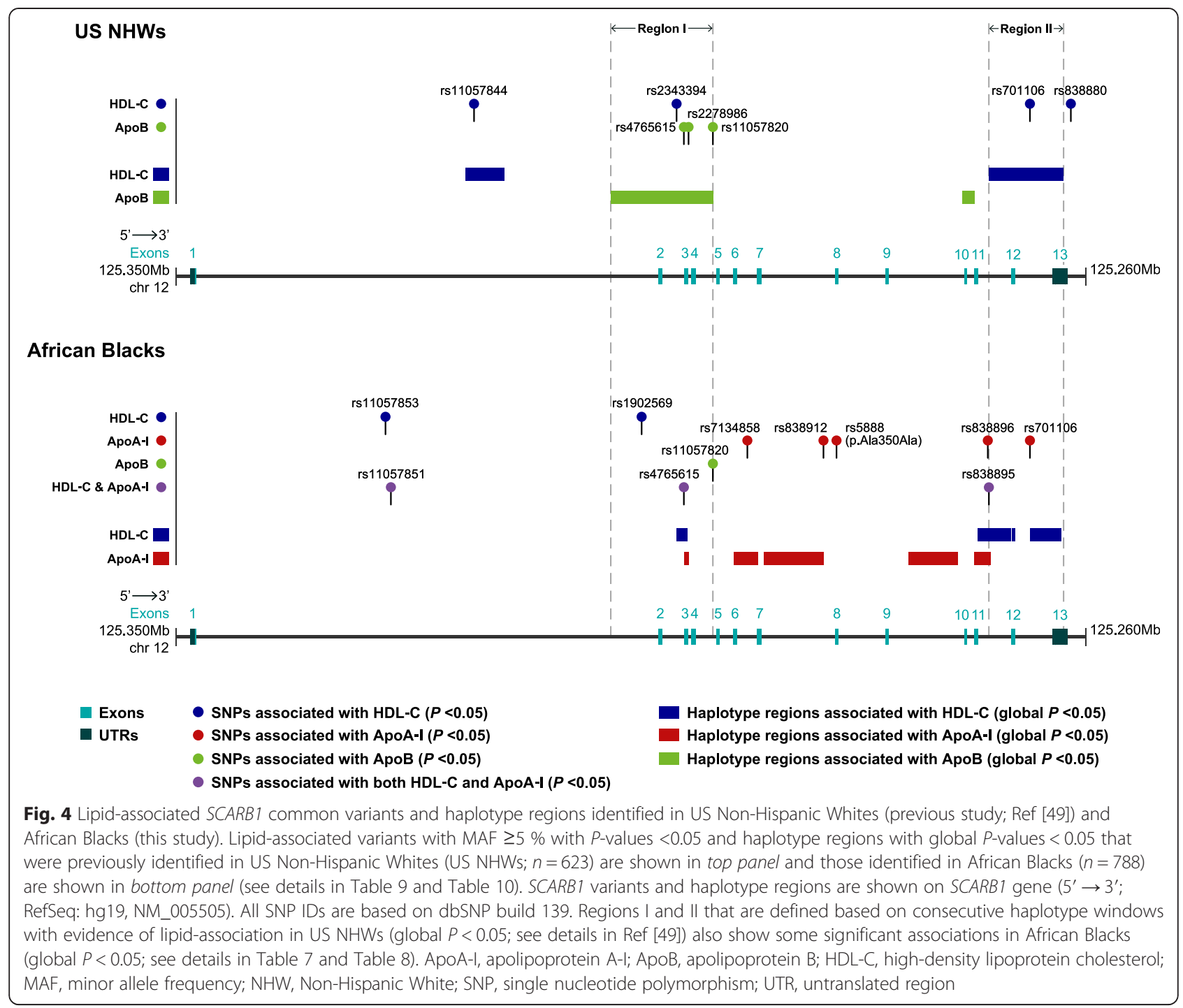

Table 3). Consistent with the gene-based results, singlesite association analyses also revealed 10 common variants nominally associated $(P<0.05)$ with HDL-C $(n=5)$ and/or ApoA-I $(n=8$; Table 4 and Fig. 2$)$. The best association signal was between rs11057851 in intron 1 and HDL-C ( $P$ $=0.0043$, FDR $=0.1465)$ followed by two associations with ApoA-I including rs7134858 in intron $6(P=0.0052$, FDR $=0.2918)$ and rs5888 (p.Ala350Ala) in exon $8(P=0.0080$, $\mathrm{FDR}=0.2918$ ). Moreover, three variants (rs11057851, rs4765615, and rs838895) exhibited evidence of associations $(P<0.05)$ with both HDL-C and ApoA-I. These findings are supported by the fact that $S C A R B 1$ appears to influence ApoA-I in addition to HDL-C [15, 17]. In our data, there was a moderate correlation between ApoA-I and HDL-C levels $\left(r^{2}=0.61\right)$.

Except for previously reported association of rs5888 (p.Ala350Ala) with lipid traits (HDL-C or LDL-C) in non-African populations $[30-34,36,37,39]$, the remaining nine associations observed in this study with the lipid traits (HDL-C and/or ApoA-I levels) in general population are novel and await replication in independent African or African-derived populations. Two of these nine SNPs have previously been shown to have differential effects on cholesterol levels in response to statin (rs4765615) [50] or on HDL-C/TG levels in response to estradiol in post-menopausal women (rs838895) [51]. Another variant (rs838896) was found to be associated with decreased SCARB1 expression in liver [51]. Although the latter SNP was not associated with a low RegulomeDB score $(<3)$, we cannot rule out the possibility that it might be affecting the $S C A R B 1$ expression in a tissue-specific manner.

The haplotype analysis revealed evidence of significant association (global $P<0.05$ ) of 32 haplotype windows with HDL-C $(n=16)$ and/or ApoA-I $(n=21$; Table 7$)$ and nine regions harboring consecutive overlapping 
haplotype windows significantly associated with either HDL-C (4 regions) or ApoA-I (5 regions; Table 8 and Fig. 3). In addition, six variants with nominal association $(P<0.05)$ in single-site analysis were contained in seven of these nine significantly associated regions, indicating the presence of functional variants in these regions. Our findings demonstrate that haplotype analysis may provide more information than single-site analysis.

Our comparison of the single-site and haplotype association results between in African Blacks (this study) and US NHWs (previous study [49]) has revealed three variants (rs11057820, rs4765615, and rs701106; Table 9) and two regions (Regions I and II; Table 10 and Fig. 4) showing evidence of lipid-associations in both ethnic groups. However, there were differences in associated traits, and/or associated alleles or their directional effects between the two ethnic groups, which reflects the genetic heterogeneity of complex phenotypes like lipid traits among diverse populations. This phenomenon can be explained by different ancestry backgrounds associated with differences in LD structure and genetic architecture, as well as by differences in SNP-SNP, gene-gene, and gene-environment interactions. Nonetheless, the lipid associations observed across different ethnic populations provide convincing evidence that causal/functional variants are present in SCARB1 gene that deserves comprehensive sequencing and functional studies in order to confirm and further characterize the effects of its variants on lipid metabolism.

Rare variant analysis showed significant evidence of association between a group of 23 rare variants (MAF $\leq 1 \%)$ and HDL-C $(P=0.0478$; Table 5). Single-site analysis of these rare variants revealed six (including three novel ones) with effects on HDL-C, of which three also had effects on ApoA-I (Table 6). In addition, four of these six rare variants appeared to be carried by individuals with extreme HDL-C and/or ApoA-I levels (above or under the 3rd percentile). This HDL-C-associated rare variant group also included a novel variant (p70201/ chr12:125279319) that was observed in one individual with an unusually high plasma HDL-C level (above the mean $+3.5 \mathrm{SD}$ ). Our findings suggest that these rare variants might have functional relevance, thus screening of additional large African samples for these rare variants may help to establish their role in HDL-C and ApoA-I metabolism.

To date, there has been limited information concerning possible functional effects of lipid-associated SCARB1 variants, particularly for those located in non-coding regions. In fact, most of common and rare HDL-C/ApoAI-associated variants observed in the current study are non-coding and do not show strong evidence of regulatory function based on RegulomeDB database. Nonetheless, three of these HDL-C/ApoA-I-associated SCARB1 variants (rs5888 [p.Ala350Ala], rs838885, and rs838886) have been previously demonstrated to influence the SCARB1 expression [51-53]. Therefore, additional functional studies are needed and may help to determine the functional nature of the SCARB1-associated variants and those in LD with them.

Our study has revealed a number of novel findings, although we also acknowledge some limitations. SCARB1 is a large gene and we sequenced only its coding regions and exon-intron junctions and also our sequencing sample size was small. Thus, we may have missed some functional $\mathrm{LoF} /$ rare variants due to small sample size and those located in uncovered intronic regions. Moreover, consistent with generally small effect sizes of lipidassociated variants reported in the literature, most of our single-site associations reached nominal significance $(P<0.05)$ but did not survive multiple testing corrections. Only the top variant (rs11057851) associated with HDL-C yielded an FDR cut-off of $<0.20$ (FDR $=0.1465$; Table 4). Therefore, future larger studies in independent African or African-derived populations are necessary to validate all nominal associations observed in this study.

\section{Conclusions}

In conclusion, we report the first comprehensive association study of SCARB1 variants with lipid traits in a native African population, which revealed a number of novel associations in single-site and haplotype analyses. In addition, resequencing allowed us to identify 10 novel rare variants, of which four were in the group of 23 rare variants that has showed association with HDL-C levels. The SCARB1 associated common and rare variants observed in our study explained $\sim 11.09 \%$ of the variation in HDL-C levels and $\sim 8.63 \%$ of the variation in ApoA-I levels. Our findings indicate the genetic contribution of SCARB1, both common and LoF/rare variants, to interindividual lipid variation in the general African Black population, which warrants further follow-up in independent studies. Insights into the HDL-C and related lipid traits may also lead to new potential targets for CHD treatment.

\section{Additional files}

Additional file 1: Table S1. Characteristics and lipid profile of the entire sample of 788 African Blacks stratified by sex. (PDF 63 kb)

Additional file 2: Table S2. Primer sequences for 14 polymerase chain reaction (PCR) fragments and the sizes of 13 SCARB1 exons. (PDF $96 \mathrm{~kb}$ )

Additional file 3: Table S3. Characteristics of 83 SCARB1 sequence variants identified in 95 African Blacks with extreme HDL-C levels. (PDF $146 \mathrm{~kb})$

Additional file 4: Table S4. Characteristics of 10 SCARB1 novel variants identified by sequencing. (PDF 81 kb)

Additional file 5: Figure S1. Linkage disequilibrium (LD) plot of 83 SCARB1 sequence variants. Of 83 sequence variants (see the list in 
Additional file 3: Table S3), 78 were selected for genotyping. An enlarged view of the part of LD plot (A) shows the pairwise correlations $\left(r^{2}\right)$ between four variants including the two variants (shown in bold) in the same bin in our data, of which one selected for genotyping. This bin was not identified by Tagger analysis of common SCARB1 variants in the HapMap-YRI data (see Additional file 7: Table S5 and Additional file 8: Figure S3). The degree of shades and values $\left(r^{2} \times 100\right)$ in each square of LD plot represent the pairwise correlations: black indicating $r^{2}=1$, white indicating $r^{2}=0$, and shade intensity indicating $r^{2}$ between 0 and 1. LD, linkage disequilibrium; MAF, minor allele frequency; YRI, Yoruba people of Ibadan from Nigeria. (PDF $920 \mathrm{~kb}$ )

Additional file 6: Figure S2. Linkage disequilibrium (LD) plot of 32 SCARB1 common sequence variants. Enlarged view of the parts of the LD plot $(\mathbf{A}, \mathbf{B}$, and $\mathbf{C})$ show three LD bins (identified by Tagger analysis of variants with minor allele frequency (MAF) $\geq 5 \%$ using an $r^{2}$ cutoff of $0.90)$ containing more than one variant $\left(r^{2}\right.$ ranging between 0.95 and 1.0). The degree of shades and values $\left(r^{2} \times 100\right)$ in each square of $L D$ plot represent the pairwise correlations: black indicating $r^{2}=1$, white indicating $r^{2}=0$, and shade intensity indicating $r^{2}$ between 0 and $1 . L D$, linkage disequilibrium; MAF, minor allele frequency. (PDF $370 \mathrm{~kb}$ )

Additional file 7: Table S5. List of 77 SCARB1 HapMap-YRI tagSNPS. (PDF $110 \mathrm{~kb}$ )

Additional file 8: Figure S3. Linkage disequilibrium (LD) plot of 108 SCARB1 common HapMap-YRI tagSNPs. The list of 77 common HapMap-YRI tagSNPs identified by Tagger analysis of variants with minor allele frequency $\geq 5 \%$ using an $r^{2}$ cutoff of 0.80 is shown in Additional file 7 : Table 55 . The degree of shades and values $\left(r^{2} \times 100\right)$ in each square of $L D$ plot represent the pairwise correlations: black indicating $r^{2}=1$, white indicating $r^{2}=0$, and shade intensity indicating $r^{2}$ between 0 and 1.LD, linkage disequilibrium; SNP, single nucleotide polymorphism; YRI, Yoruba people of Ibadan from Nigeria. (TIFF $2642 \mathrm{~kb}$ )

Additional file 9: Table S6. Characteristics of 138 SCARB1 variants genotyped in the entire sample of 788 African Blacks. (PDF $212 \mathrm{~kb}$ )

Additional file 10: Table S7. List of 87 SCARB1 genotyped common tagSNPs. (PDF $108 \mathrm{~kb}$ )

Additional file 11: Figure S4. Linkage disequilibrium (LD) plot of 137 SCARB1 genotyped variants. The list of 87 genotyped common tagSNPs identified by Tagger analysis for variants with minor allele frequency $\geq 5 \%$ using an $r^{2}$ cutoff of 0.90 is shown in Additional file 10: Table S7. The degree of shades and values $\left(r^{2} \times 100\right)$ in each square of LD plot represent the pairwise correlations: black indicating $r^{2}=1$, white indicating $r^{2}=0$, and shade intensity indicating $r^{2}$ between 0 and 1.LD, linkage disequilibrium; SNP, single nucleotide polymorphism. (TIFF $3095 \mathrm{~kb}$ )

Additional file 12: Figure S5. Location and minor allele frequency (MAF) distributions of 137 SCARB1 genotyped variants. Details for each variant are shown in Additional file 9: Table S6. MAF, minor allele frequency; UTR, untranslated region. (TIFF $324 \mathrm{~kb}$ )

Additional file 13: Table S8. Covariates used for the statistical analysis of lipid variables. (PDF $78 \mathrm{~kb}$ )

Additional file 14: Table S9. Single-site association results for 136 SCARB1 genotyped variants with HDL-C. (PDF $689 \mathrm{~kb}$ )

Additional file 15: Table S10. Single-site association results for 136 SCARB1 genotyped variants with ApoA-I. (PDF $686 \mathrm{~kb}$ )

Additional file 16: Table S11. Haplotype association results for 136 SCARB1 genotyped variants for HDL-C and ApoA-I. (PDF $257 \mathrm{~kb}$ )

Additional file 17: Table S12. Summary of RegulomeDB scores of 153 SCARB1 variants. (PDF $95 \mathrm{~kb}$ )

Additional file 18: Table S13. RegulomeDB scores and functional assignments of 153 SCARB1 variants. (PDF $149 \mathrm{~kb}$ )

\section{Abbreviations}

ApoA-l: Apolipoprotein A-l; ApoB: Apolipoprotein B; BP: Base pair; CE: Cholesteryl esters; CHD: Coronary heart disease; FDR: False discovery rate; GWAS: Genome-wide association studies; HDL-C: High-density lipoprotein cholesterol; HWE: Hardy-Weinberg equilibrium; indel: Insertion and deletion variation; KB: Kilobase pair; LD: Linkage disequilibrium; LDL-C: Low-density lipoprotein cholesterol; LoF: Low-frequency; MAF: Minor allele frequency; NHW: Non-Hispanic White; PCR: Polymerase chain reaction; QC: Quality controls; RCT: Reverse cholesterol transport; SCARB1: Scavenger receptor class B member 1; SD: Standard deviation; SKAT-O: An optimal sequence kernel association test; SNP: Single nucleotide polymorphism;

SNV: Singlenucleotide variation; TG: Triglycerides; UTR: Untranslated region; VEGAS: Versatile gene-based association study; VLDL-C: Very low-density lipoprotein cholesterol; YRI: Yoruba people of Ibadan from Nigeria.

\section{Competing interests}

The authors declare that they have no competing interests.

\section{Authors' contributions}

Conceive and design the experiments: FYD, MIK Perform the experiments: VN FYD Analyze the data: VN, XW, DP, ZHR, MMB, FYD, MIK Contribute reagents/ materials/analysis tools: CHB, MIK Write the paper: VN, FYD, MIK Provide critical revisions: XW, DP, ZHR, CHB, MMB Interpret the results: VN, XW, DP, ZHR, CHB, $M M B, F Y D, M I K$ All authors read and approved the final manuscript.

\section{Acknowledgements}

This study was supported by the National Heart, Lung and Blood Institute [NHLBI] (grant numbers HL044413 to C.H.B. and HL084613 to M.I.K.).

\section{Author details}

'Department of Human Genetics, Graduate School of Public Health, University of Pittsburgh, 130 DeSoto Street, Pittsburgh, PA 15261, USA.

2Department of Epidemiology, Graduate School of Public Health, University of Pittsburgh, 130 DeSoto Street, Pittsburgh, PA 15261, USA.

Received: 9 April 2015 Accepted: 30 October 2015

Published online: 12 November 2015

\section{References}

1. National Cholesterol Education Program. Third report of the national cholesterol education program (NCEP) expert panel on detection, evaluation, and treatment of high blood cholesterol in adults (adult treatment panel III) final report. Circulation. 2002;106(25):3143-421.

2. World Health Organization. The 10 leading causes of death in the world, 2000 and 2012. In: The top 10 causes of death. Media centre, World Health Organization, 2014. Available from http://www.who.int/mediacentre/ factsheets/fs310/en/. (accessed 30 April 2015).

3. O'Connell DL, Heller RF, Roberts DC, Allen JR, Knapp JC, Steele PL, et al. Twin study of genetic and environmental effects on lipid levels. Genet Epidemiol. 1988;5(5):323-41.

4. Kathiresan S, Willer CJ, Peloso GM, Demissie S, Musunuru K, Schadt EE, et al. Common variants at 30 loci contribute to polygenic dyslipidemia. Nat Genet. 2009:41(1):56-65.

5. Teslovich TM, Musunuru K, Smith AV, Edmondson AC, Stylianou IM, Koseki $\mathrm{M}$, et al. Biological, clinical and population relevance of 95 loci for blood lipids. Nature. 2010;466(7307):707-13.

6. Consortium GLG, Willer CJ, Schmidt EM, Sengupta S, Peloso GM, Gustafsson $\mathrm{S}$, et al. Discovery and refinement of loci associated with lipid levels. Nat Genet. 2013;45(11):1274-83

7. Khetarpal SA, Rader DJ. Genetics of lipid traits: Genome-wide approaches yield new biology and clues to causality in coronary artery disease. Biochim Biophys Acta. 2014;1842(10):2010-20. doi:10.1016/j.bbadis.2014.06.007.

8. Kuivenhoven JA, Hegele RA. Mining the genome for lipid genes. Biochim Biophys Acta. 2014;1842(10):1993-2009. doi:10.1016/j.bbadis.2014.04.028.

9. Cohen JC, Kiss RS, Pertsemlidis A, Marcel YL, McPherson R, Hobbs HH. Multiple rare alleles contribute to low plasma levels of HDL cholesterol. Science. 2004;305(5685):869-72.

10. Frikke-Schmidt R, Nordestgaard BG, Jensen GB, Tybjaerg-Hansen A. Genetic variation in $A B C$ transporter $A 1$ contributes to $H D L$ cholesterol in the general population. J Clin Invest. 2004;114(9):1343-53.

11. Peloso GM, Auer PL, Bis JC, Voorman A, Morrison AC, Stitziel NO, et al, Association of low-frequency and rare coding-sequence variants with blood lipids and coronary heart disease in 56,000 whites and blacks. Am J Hum Genet. 2014;94(2):223-32.

12. Rye KA, Barter PJ. Cardioprotective functions of HDLs. J Lipid Res. 2014;55(2):168-79. 
13. Rosenson RS, Brewer Jr HB, Davidson WS, Fayad ZA, Fuster V, Goldstein J, et al. Cholesterol efflux and atheroprotection: advancing the concept of reverse cholesterol transport. Circulation. 2012;125(15):1905-19.

14. Trigatti $B L$, Krieger M, Rigotti $A$. Influence of the $\mathrm{HDL}$ receptor SR-BI on lipoprotein metabolism and atherosclerosis. Arterioscler Thromb Vasc Biol. 2003;23(10):1732-8.

15. Kozarsky KF, Donahee MH, Rigotti A, lqbal SN, Edelman ER, Krieger M. Overexpression of the HDL receptor SR-BI alters plasma $\mathrm{HDL}$ and bile cholesterol levels. Nature. 1997;387(6631):414-7.

16. Wang N, Arai T, Ji Y, Rinninger F, Tall AR. Liver-specific overexpression of scavenger receptor $B I$ decreases levels of very low density lipoprotein ApoB, low density lipoprotein $A p o B$, and high density lipoprotein in transgenic mice. J Biol Chem. 1998;273(49):32920-6.

17. Ueda Y, Royer L, Gong E, Zhang J, Cooper PN, Francone O, et al. Lower plasma levels and accelerated clearance of high density lipoprotein (HDL) and non-HDL cholesterol in scavenger receptor class B type I transgenic mice. J Biol Chem. 1999;274(11):7165-71.

18. Out R, Kruijt JK, Rensen PC, Hildebrand RB, de Vos P, Van Eck M, et al. Scavenger receptor $\mathrm{BI}$ plays a role in facilitating chylomicron metabolism. J Biol Chem. 2004;279(18):18401-6.

19. Out R, Hoekstra M, de Jager SC, de Vos P, van der Westhuyzen DR, Webb $N R$, et al. Adenovirus-mediated hepatic overexpression of scavenger receptor class B type I accelerates chylomicron metabolism in C57BL/6 J mice. J Lipid Res. 2005;46(6):1172-81.

20. Van Eck M, Hoekstra M, Out R, Bos IS, Kruijt JK, Hildebrand RB, et al. Scavenger receptor BI facilitates the metabolism of VLDL lipoproteins in vivo. J Lipid Res. 2008;49(1):136-46.

21. Wiersma H, Nijstad N, Gautier T, lqbal J, Kuipers F, Hussain MM, et al. Scavenger receptor $\mathrm{Bl}$ facilitates hepatic very low density lipoprotein production in mice. J Lipid Res. 2010;51(3):544-53.

22. Trigatti B, Rigotti A, Krieger M. The role of the high-density lipoprotein receptor SR-BI in cholesterol metabolism. Curr Opin Lipidol. 2000;11(2):123-31.

23. Rhainds $D$, Brissette $L$. The role of scavenger receptor class $B$ type I (SR-BI) in lipid trafficking. defining the rules for lipid traders. Int J Biochem Cell Biol. 2004;36(1):39-77

24. Rigotti A, Trigatti BL, Penman M, Rayburn H, Herz J, Krieger M. A targeted mutation in the murine gene encoding the high density lipoprotein $(\mathrm{HDL})$ receptor scavenger receptor class B type I reveals its key role in HDL metabolism. Proc Natl Acad Sci U S A. 1997;94(23):12610-5.

25. Varban ML, Rinninger F, Wang N, Fairchild-Huntress V, Dunmore JH, Fang Q, et al. Targeted mutation reveals a central role for SR-BI in hepatic selective uptake of high density lipoprotein cholesterol. Proc Natl Acad Sci U S A. 1998;95(8):4619-24.

26. Trigatti B, Rayburn H, Vinals M, Braun A, Miettinen H, Penman M, et al. Influence of the high density lipoprotein receptor SR-BI on reproductive and cardiovascular pathophysiology. Proc Natl Acad Sci U S A 1999;96(16):9322-7.

27. Ji Y, Wang N, Ramakrishnan R, Sehayek E, Huszar D, Breslow JL, et al. Hepatic scavenger receptor Bl promotes rapid clearance of high density lipoprotein free cholesterol and its transport into bile. J Biol Chem. 1999:274(47):33398-402.

28. Brunham LR, Tietjen I, Bochem AE, Singaraja RR, Franchini PL, Radomski C, et al. Novel mutations in scavenger receptor Bl associated with high HDL cholesterol in humans. Clin Genet. 2011;79(6):575-81.

29. Vergeer M, Korporaal SJ, Franssen R, Meurs I, Out R, Hovingh GK, et al. Genetic variant of the scavenger receptor BI in humans. N Engl J Med. 2011;364(2):136-45.

30. Acton S, Osgood D, Donoghue M, Corella D, Pocovi M, Cenarro A, et al. Association of polymorphisms at the SR-BI gene locus with plasma lipid levels and body mass index in a white population. Arterioscler Thromb Vasc Biol. 1999;19(7):1734-43.

31. Hong SH, Kim YR, Yoon YM, Min WK, Chun SI, Kim JQ. Association between Haelll polymorphism of scavenger receptor class B type I gene and plasma HDL-cholesterol concentration. Ann Clin Biochem. 2002:39(Pt 5):478-81.

32. Osgood D, Corella D, Demissie S, Cupples LA, Wilson PW, Meigs JB, et al. Genetic variation at the scavenger receptor class B type I gene locus determines plasma lipoprotein concentrations and particle size and interacts with type 2 diabetes: the framingham study. J Clin Endocrinol Metab. 2003;88(6):2869-79.

33. Tai ES, Adiconis X, Ordovas JM, Carmena-Ramon R, Real J, Corella D, et al. Polymorphisms at the SRBI locus are associated with lipoprotein levels in subjects with heterozygous familial hypercholesterolemia. Clin Genet. 2003;63(1):53-8

34. Morabia A, Ross BM, Costanza MC, Cayanis E, Flaherty MS, Alvin GB, et al. Population-based study of SR-BI genetic variation and lipid profile. Atherosclerosis. 2004;175(1):159-68.

35. Richard E, von Muhlen D, Barrett-Connor E, Alcaraz J, Davis R, McCarthy J. J. Modification of the effects of estrogen therapy on $\mathrm{HDL}$ cholesterol levels by polymorphisms of the HDL-C receptor, SR-BI: the Rancho Bernardo Study. Atherosclerosis. 2005;180(2):255-62.

36. Boekholdt SM, Souverein OW, Tanck MW, Hovingh GK, Kuivenhoven JA, Peters Rl, et al. Common variants of multiple genes that control reverse cholesterol transport together explain only a minor part of the variation of HDL cholesterol levels. Clin Genet. 2006;69(3):263-70.

37. Roberts CG, Shen H, Mitchell BD, Damcott CM, Shuldiner AR, Rodriguez A. Variants in scavenger receptor class B type I gene are associated with HDL cholesterol levels in younger women. Hum Hered. 2007;64(2):107-13.

38. Tanaka T, Delgado-Lista J, Lopez-Miranda J, Perez-Jimenez F, Marin C, Perez-Martinez $P$, et al. Scavenger receptor class B type I (SCARB1) c.1119C > T polymorphism affects postprandial triglyceride metabolism in men. J Nutr. 2007;137(3):578-82.

39. Cerda A, Genvigir FD, Arazi SS, Hirata MH, Dorea EL, Bernik MM, et al. Influence of SCARB1 polymorphisms on serum lipids of hypercholesterolemic individuals treated with atorvastatin. Clin Chim Acta. 2010;411(9-10):631-7.

40. Manichaikul A, Naj AC, Herrington D, Post W, Rich SS, Rodriguez A. Association of SCARB1 variants with subclinical atherosclerosis and incident cardiovascular disease: the multi-ethnic study of atherosclerosis. Arterioscler Thromb Vasc Biol. 2012;32(8):1991-9.

41. Bunker $\mathrm{CH}$, Ukoli FA, Okoro Fl, Olomu AB, Kriska AM, Huston SL, et al. Correlates of serum lipids in a lean black population. Atherosclerosis. 1996;123(1-2):215-25.

42. Friedewald WT, Levy RI, Fredrickson DS. Estimation of the concentration of low-density lipoprotein cholesterol in plasma, without use of the preparative ultracentrifuge. Clin Chem. 1972;18(6):499-502.

43. Barrett JC, Fry B, Maller J, Daly MJ. Haploview: analysis and visualization of LD and haplotype maps. Bioinformatics. 2005;21(2):263-5.

44. Liu JZ, MCRae AF, Nyholt DR, Medland SE, Wray NR, Brown KM, et al. A versatile gene-based test for genome-wide association studies. Am J Hum Genet. 2010;87(1):139-45.

45. Benjamini Y, Hochberg Y. Controlling the false discovery rate: a practical and powerful approach to multiple testing. J R Stat Soc Ser B (Stat Methodol). 1995;57:289-300.

46. Lee $\mathrm{S}, \mathrm{Wu} \mathrm{MC}$, Lin X. Optimal tests for rare variant effects in sequencing association studies. Biostatistics. 2012;13(4):762-75.

47. Cao G, Garcia CK, Wyne KL, Schultz RA, Parker KL, Hobbs HH. Structure and localization of the human gene encoding SR-BI/CLA-1. Evidence for transcriptional control by steroidogenic factor 1. J Biol Chem. 1997;272(52):33068-76.

48. Boyle AP, Hong EL, Hariharan M, Cheng Y, Schaub MA, Kasowski M, et al. Annotation of functional variation in personal genomes using RegulomeDB. Genome Res. 2012;22(9):1790-7.

49. Niemsiri V, Wang X, Pirim D, Radwan ZH, Hokanson JE, Hamman RF, et al. Impact of genetic variants in human scavenger receptor class B type I (SCARB1) on plasma lipid traits. Circ Cardiovasc Genet. 2014;7(6):838-47.

50. Peters BJ, Pett $\mathrm{H}$, Klungel OH, Stricker BH, Psaty BM, Glazer NL, et al. Genetic variability within the cholesterol lowering pathway and the effectiveness of statins in reducing the risk of MI. Atherosclerosis. 2011;217(2):458-64.

51. Chiba-Falek O, Nichols M, Suchindran S, Guyton J, Ginsburg GS, Barrett-Connor $E$, et al. Impact of gene variants on sex-specific regulation of human Scavenger receptor class B type 1 (SR-BI) expression in liver and association with lipid levels in a population-based study. BMC Med Genet. 2010;11:9.

52. Constantineau J, Greason E, West M, Filbin M, Kieft JS, Carletti MZ, et al. A synonymous variant in scavenger receptor, class $B$, type I gene is associated with lower SR-BI protein expression and function. Atherosclerosis. 2010;210(1):177-82.

53. Cerda A, Genvigir FD, Rodrigues AC, Willrich MA, Dorea EL, Bernik MM, et al. Influence of polymorphisms and cholesterol-lowering treatment on SCARB1 mRNA expression. J Atheroscler Thromb. 2011;18(8):640-51. 